\title{
Maintenance of Metabolic Plasticity Despite Relaxed Selection in a Long-Term Evolution Experiment with Escherichia coli
}

\author{
Nkrumah A. Grant, ${ }^{1,2,3^{*}}$ Rohan Maddamsetti, ${ }^{4}$ and Richard E. Lenski ${ }^{1,2,3}$
}

1. BEACON Center for the Study of Evolution in Action, Michigan State University, East Lansing, Michigan 48824; 2. Department of Microbiology and Molecular Genetics, Michigan State University, East Lansing, Michigan 48824; 3. Program in Ecology, Evolutionary Biology and Behavior, Michigan State University, East Lansing 48824; 4. Department of Biomedical Engineering, Duke University, Durham, North Carolina 27708.

* Corresponding author; e-mail: nkrumah.grant88@gmail.com 
2 ABSTRACT: Traits that are unused in a given environment are subject to processes that tend to erode

3 them, leading to reduced fitness in other environments. Although this general tendency is clear,

4 we know much less about why some traits are lost while others are retained, and about the roles of

5 mutation and selection in generating different responses. We addressed these issues by examining

6 populations of a facultative anaerobe, Escherichia coli, that have evolved for $>30$ years in the

7 presence of oxygen, with relaxed selection for anaerobic growth and the associated metabolic

8 plasticity. We asked whether evolution led to the loss, improvement, or maintenance of anaerobic

9 growth, and we analyzed gene expression and mutational datasets to understand the outcomes. We

10 identified genomic signatures of both positive and purifying selection on aerobic-specific genes,

11 while anaerobic-specific genes showed clear evidence of relaxed selection. We also found parallel

12 evolution at two interacting loci that regulate anaerobic growth. We competed the ancestor and

13 evolved clones from each population in an anoxic environment, and we found that anaerobic

14 fitness had not decayed, despite relaxed selection. In summary, relaxed section does not

15 necessarily reduce an organism's fitness in other environments. Instead, the genetic architecture

16 of the traits under relaxed selection and their correlations with traits under positive and purifying

17 selection may sometimes determine evolutionary outcomes.

19 Keywords: bacteria, correlated responses, genetic architecture, metabolism, mutation, pleiotropy 


\section{Introduction}

"[I]f man goes on selecting, and thus augmenting, any peculiarity, he will almost certainly unconsciously modify other parts of the structure, owing to the mysterious laws of the correlation of growth." — Charles Darwin, On the Origin of Species, 1859

27 Organisms seldom experience static conditions. Instead, they typically experience fluctuations in

28 both their external environments and internal states. Organisms have adapted to these fluctuations

29 by evolving a variety of mechanisms to maintain homeostasis and survive, that is to be

30 phenotypically robust, in the face of environmental and genetic perturbations (Lenski et al. 2006;

31 Frankel et al. 2010; Fraser and Schadt 2010; Siegal and Leu 2014). One mechanism to maintain

32 homeostasis is metabolic plasticity, by which we mean the innate capacity to change metabolic

33 fluxes in response to changes in the environment (Jia et al. 2019). Metabolic plasticity is controlled

34 by genes, the expression of which is coupled to one or more environmental signals (Paudel and

35 Quaranta 2019). Bacillus subtilis, for example, produces metabolically dormant endospores when

36 cells are starved for nutrients (Setlow 2006). Metabolic plasticity can sometimes go awry, such as

37 when cancer cells perform glycolysis instead of oxidative phosphorylation (Warburg 1956) to

38 generate ATP and synthesize biomass, promoting tumorigenesis and metastatic potential (Payen

39 et al. 2016). More generally, metabolic plasticity determines the environmental conditions in

40 which an organism can survive and grow. However, much remains unknown about the

41 mechanisms underlying metabolic plasticity and the resulting phenotypic robustness, and how

42 these mechanisms and robustness have evolved and continue to evolve (Siegal and Leu 2014;

43 Nijhout et al. 2017). 
Experimental evolution with microorganisms has proven to be a powerful way to study the evolutionary process (Elena and Lenski 2003; Lenski 2017; Van den Bergh et al. 2018). These experiments typically maintain relatively simple and constant conditions, which places many traits,

47 including metabolic plasticity, under relaxed selection. Accordingly, many such studies have shown losses of functions owing to antagonistic pleiotropy (including the cost of expressing unneeded traits), mutation accumulation in unused genes, or both (Cooper and Lenski 2000; Cooper et al. 2001b; Maughan et al. 2009; Leiby and Marx 2014; Lamrabet et al. 2019). However, unneeded traits may sometimes be maintained and even improved if the underlying genes serve

52 multiple purposes, such that the unneeded trait is genetically correlated with a trait under positive 53 selection (Bennett et al. 1990). The latter scenario has been termed buttressing pleiotropy (Lahti

54 et al. 2009). Computational models and experiments with artificial organisms suggest that

55 buttressing pleiotropy readily occurs when new functions evolve by building upon existing 56 functions (Wagner and Mezey 2000; Lenski et al. 2003; Ostrowski et al. 2015). However, the extent of buttressing pleiotropy in biological systems remains unclear and has been little studied.

To study how metabolic plasticity evolves under relaxed selection, we analyzed both

59 phenotypic performance and genetic changes in Escherichia coli populations from the long-term

60 evolution experiment (LTEE). The 12 LTEE populations have been evolving independently in a

61 glucose-limited minimal medium with constant aeration for more than 70,000 generations (Lenski

62 et al. 1991; Good et al. 2017). Samples are frozen every 500 generations, generating a "frozen

63 fossil record" from which bacteria can be revived for genetic and phenotypic analyses, including

64 measuring their fitness in the LTEE environment and other environments that differ in various

65 respects. By 50,000 generations the populations were, on average, about $70 \%$ more fit than their

66 common ancestor in the LTEE environment (Wiser et al. 2013). Most of that improvement 
67 occurred in the first 10,000 generations, but the populations have continued to improve at slower

68 rates throughout the experiment (Lenski et al. 2015; Lenski 2017). The bacteria experienced

69 relaxed selection for anaerobic growth during the long duration of their evolution in the strictly

70 oxic LTEE environment. To date, several hundred clones and more than 1400 mixed population

71 samples have been sequenced (Tenaillon et al. 2016; Good et al. 2017), providing material for

72 examining the coupling between aerobic and anaerobic fitness and the underlying genetic changes.

73 Six LTEE populations evolved hypermutability during the experiment (Tenaillon et al. 2016; Good

74 et al. 2017), which should increase the rate at which unused genes accumulate mutations and

75 unused functions decay over time (Cooper and Lenski 2000; Leiby and Marx 2014). The

76 hypermutable lineages exhibited $\sim 100$-fold increases in their point-mutation rate (Sniegowski et

77 al. 1997; Wielgoss et al. 2013), but the rate of fitness gain in these lineages increased by only a

78 few percent relative to other populations (Wiser et al. 2013; Lenski et al. 2015). This difference

79 facilitates disentangling the effects of antagonistic pleiotropy from those of mutation accumulation

80 on the phenotypic and genomic consequences of relaxed selection.

81 In addition to the strength of the LTEE model system for asking evolutionary questions, $E$.

82 coli is an excellent model for studying the evolution of metabolic plasticity. As a facultative

83 anaerobe, E. coli is able to survive, grow, and reproduce in both oxic and anoxic environments

84 (Unden et al. 1994). This plasticity allows E. coli to inhabit diverse environments that vary in

85 oxygen availability, from the gastrointestinal tracts of mammals (and some birds and reptiles) to

86 freshwater and soil environments (Gordon and Cowling 2003). The molecular control of this

87 plasticity is well-understood, and two global regulatory systems play critical roles (Gunsalus and

88 Park 1994; Unden and Bongaerts 1997). The Fumarate and Nitrate Reductase protein (FNR),

89 encoded by the $f n r$ gene, is a transcription factor that directly senses oxygen (Gunsalus and Park 
90 1994; Kang et al. 2005). The Anoxic Respiratory Control system, encoded by $\operatorname{arc} A$ and $\operatorname{arcB}$, is a

91 canonical two-component regulatory system that responds to oxygen and the redox status of the

92 cell, as shown in Figure 1 (Iuchi and Lin 1988; Gunsalus and Park 1994; Unden and Bongaerts

93 1997). The transcriptional control conferred by the $f n r$ and $\operatorname{arc} A B$ regulons allows $E$. coli cells to

94 commit physiologically to either aerobic or anaerobic metabolism, depending on oxygen

95 availability. Mutations in either regulon may disrupt this control (Melville and Gunsalus 1990). In

96 particular, some mutations in $\operatorname{arc} A B$ have been shown to alter metabolism by causing the

97 constitutive expression of genes that would normally be responsive to oxygen concentration and

98 internal redox balance (Iuchi and Lin 1988; Saxer et al. 2014).

In this study, we sought to determine whether relaxed selection during 50,000 generations

100 of strictly aerobic growth led to the loss of anaerobic performance and the associated metabolic

101 plasticity. Alternatively, genetic correlations between aerobic and anaerobic physiology might

102 have favored the maintenance or even improvement of anaerobic metabolism during evolution in

103 the oxic environment of the LTEE. This alternative, if observed, might reflect the fact that

104 anaerobic metabolism evolved more than 2 billion years before the origin of aerobic metabolism

105 (Müller 1977; Soo et al. 2017), such that the genetic and biochemical networks underpinning

106 metabolism in these conditions might be tightly coupled. It could also indicate the biochemical

107 promiscuity of many proteins involved in metabolism (Nam et al. 2012). In any case, we tested

108 the phenotypic correlation in performance by measuring the fitness of the evolved LTEE clones

109 against a marked ancestor in oxic and anoxic environments. In fact, anaerobic growth capacity was

110 not only maintained under relaxed selection, but in some cases actually improved. Fitness gains

111 were seen even in some populations that evolved high mutation rates - a change that promoted 
112 mutation accumulation in anaerobic-specific genes - and despite mutations affecting the Arc AB

113 regulon.

114 Our results highlight the importance of understanding how a trait is encoded in a genetic

115 network, and how that encoding affects its evolutionary fate in the absence of direct selection. A

116 better understanding of the mechanisms that maintain anaerobic growth may also help synthetic

117 biologists design more robust systems by exploiting pleiotropy (Stirling et al. 2017; Blazejewski

118 et al. 2019; Geng et al. 2019). In addition, this work may provide a framework for better predicting

119 how the genetic encoding of traits affects an organism's evolutionary potential, including in

120 response to ecological challenges such as those caused by climate change.

\section{Materials and Methods}

126 The LTEE consists of 12 E. coli populations derived from a common ancestral strain, REL606

127 (Lenski et al. 1991). Six populations descend directly from REL606. The other six descend from 128 REL607, which differs from REL606 by two mutations that are selectively neutral under LTEE 129 conditions (Tenaillon et al. 2016). One is a point mutation in the araA gene that allows REL607

130 to utilize arabinose, and the second is an inadvertent secondary mutation of no consequence. The 131 mutation in araA provides a phenotypic marker that can be readily scored in the competition assays 132 used to measure relative fitness. When plated on tetrazolium arabinose (TA) indicator agar plates, 133 REL606 and its direct descendants form red colonies, whereas REL607 and its descendants form 134 white colonies. The ability to freeze and revive viable strains has allowed the establishment of the 
135 frozen fossil record that includes samples from all 12 populations at 500-generation intervals. This

136 record allows both genotypic and phenotypic changes to be quantified retrospectively. In this

137 study, we examined ancestral and evolved clones that were frozen at generations 2,000, 10,000

138 and 50,000 (supplemental PDF, Table S1).

\section{Culture Conditions}

142 Unless noted otherwise, we grew strains in oxic and anoxic environments in $10 \mathrm{~mL}$ of Davis

143 Mingioli minimal salts medium supplemented with $25 \mu \mathrm{g} / \mathrm{mL}$ glucose (DM25). We prepared

144 anaerobic media by boiling $500-\mathrm{mL}$ batches for $25 \mathrm{~min}$ while sparging in nitrogen gas using a

145 hypodermic needle inserted through a butyl rubber stopper. Cultures were incubated at $37^{\circ} \mathrm{C}$ in

$14650-\mathrm{mL}$ Erlenmeyer flasks, with orbital shaking at $120 \mathrm{rpm}$ in the oxic but not the anoxic

147 environment. These conditions are the same as those used during the LTEE, except for the absence

148 of oxygen and shaking during anaerobic growth.

152 We revived frozen clones isolated from each LTEE population at generations 2,000, 10,000 and

15350,000 . We used clones for which whole-genome sequences are available (Tenaillon et al. 2016).

154 The notation Ara-1 to Ara-6 denotes clones descended from the ancestral strain REL606, while 155 Ara +1 to Ara +6 are derived from REL607. We excluded from these assays the 50,000-generation 156 clones from three populations (Ara-2, Ara-3, Ara+6), as their evolved phenotypes make the 157 assays unreliable (Wiser et al. 2013). We also excluded the clones sampled from Ara+6 at both 
158 earlier time-points because their growth was erratic in the anoxic environment. Clones were

159 revived by inoculating $15 \mu \mathrm{L}$ of thawed frozen stock into $10 \mathrm{~mL}$ of Luria-Bertani (LB) broth, and

160 they were grown at $37^{\circ} \mathrm{C}$ in an orbital shaker under atmospheric conditions for $24 \mathrm{~h}$. We then

161 diluted each competitor 1:10,000 into DM25 medium for a preconditioning step that depended on

162 whether the assay would be performed in the oxic or anoxic environment. For the former, the

163 competitors were preconditioned in DM25 under the standard LTEE conditions. For the latter, the

164 competitors were preconditioned in anaerobic DM25 in an anaerobic chamber under a 95\%-

$165 \mathrm{~N}_{2}: 5 \%-\mathrm{H}_{2}$ atmosphere. Each preconditioned competitor was then diluted 1:200 into a flask

166 containing the relevant medium under the appropriate atmosphere, and the competition ran for one

167 day, during which time the combined population grew 100-fold. The one-day competition assays

168 encompassed the same lag, exponential growth, and stationary phases as populations experienced

169 during the LTEE (Lenski et al. 1991; Vasi et al. 1994). We competed the Ara ${ }^{-}$evolved clones

170 against REL607 and the $\mathrm{Ara}^{+}$evolved clones against REL606. Competitions were replicated five-

171 fold for all clones across all generations and in both environments. Owing to technical errors, only

172 four replicates yielded data for: Ara-1 at 2,000 generations, and Ara +1 and Ara +4 at 50,000

173 generations, in the oxic environment; and Ara-2 and Ara +2 at 2,000 generations, Ara +1 at 10,000

174 generations, and Ara-1 at 50,000 generations in the anoxic environment. We calculated the fitness

175 of an evolved clone relative to the ancestral competitor as the ratio of their growth rates realized

176 during the competition assay (Lenski et al. 1991; Wiser et al. 2013). 
180 The genomes of the clones used in this study were previously sequenced (Tenaillon et al. 2016).

181 We used an online tool (http://barricklab.org/shiny/LTEE-Ecoli/) to identify all of the mutations

182 discovered specifically in the $f n r$ and $\operatorname{arc} A B$ genes. To identify genes regulated in response to

183 oxygen levels, Salmon et al. (2005) performed a Bayesian analysis of gene-expression data

184 obtained for $E$. coli $\mathrm{K} 12$ in oxic and anoxic environments. We then used the set of genes from that

185 study that showed differential expression between oxic and anoxic conditions at a posterior

186 probability greater than 99\%. Those genes were mapped onto the REL606 reference genome using

187 the OMA (Altenhoff et al. 2018) and EcoCyc (Keseler et al. 2017) databases. We call genes that

188 are upregulated under oxic conditions "aerobic-specific genes," and those upregulated under

189 anoxic conditions "anaerobic-specific genes." We performed binomial tests to compare the

190 numbers of mutations in the LTEE-derived genomes in aerobic- and anaerobic-specific genes to a

191 null expectation based on the summed length of genes in the two gene sets. That analysis was

192 conducted using an R script called aerobic-anaerobic-genomics.R.

193 In addition, the LTEE metagenomics dataset includes mutations found by sequencing

194 whole-population samples for all 12 populations through 60,000 generations. These mutations

195 were downloaded from https://github.com/benjaminhgood/LTEE-metagenomic/. The data were

196 reformatted (*.csv) and analyzed using an R script called aerobic-anaerobic-metagenomics.R. In

197 brief, the cumulative number of mutations observed in each population was plotted, after

198 normalizing by gene length, for various categories of mutations. In particular, we examined subsets

199 of these data based on mutation type (nonsynonymous, synonymous, and all others including

200 indels, nonsense, and structural variants) and by function (occurring in the aerobic- or anaerobic-

201 specific genes). To generate a null expectation, we chose 10,000 random sets of genes (with the

202 same cardinality as the aerobic- and anaerobic-specific genes in the specific comparison), and the 
203 cumulative number of mutations in each set was calculated. The proportion of replicates in which

204 the cumulative number of mutations in the random set was larger than the corresponding number

205 in the aerobic- or anaerobic-specific gene set was used as an empirical $p$-value for testing statistical

206 significance. For visualization, the relevant figures show only the middle $95 \%$ of the cumulative

207 mutations for 1,000 (rather than 10,000) random sets. Statistical analyses were performed in R

208 (version 3.5.0; 2018-04-23). Datasets and R analysis scripts are available on the Dryad Digital

209 Repository (DOI pending publication).

213 We used the Cytoscape platform (Shannon et al. 2003) to visualize the biomolecular interaction

214 network between aerobic- and anaerobic-specific genes. In brief, we imported our gene sets into

215 Cytoscape and then used the software to query the STRING database (Szklarczyk et al. 2017),

216 which includes empirically known and computationally predicted protein-protein interactions.

217 Interactions are evaluated using seven lines of evidence, and a score is assigned to each

218 (Szklarczyk et al. 2017). The STRING software then computes a combined "confidence score" for

219 each interaction, which is effectively the likelihood that the interaction truly exists given the

220 evidence. We constructed our network of the aerobic- and anaerobic-specific genes showing only

221 those interactions with confidence scores greater than $70 \%$, a threshold considered "high" by the 222 database curators. 


\section{Results}

Signatures of Selection on Aerobic-and Anaerobic-specific Genes in the LTEE

228 We hypothesized that a subset of the aerobic-specific genes experienced positive selection to

229 acquire mutations that better adapt the bacteria to the LTEE environment. By contrast, we expect

230 that many anaerobic-specific genes were under relaxed selection in the LTEE. We tested these

231 predictions using the genomic and metagenomic datasets spanning 50,000 and 60,000 generations,

232 respectively (Tenaillon et al. 2016; Good et al. 2017). At various times, 6 of the 12 LTEE

233 populations evolved roughly 100 -fold higher point-mutation rates than the ancestral strain

234 (Sniegowski et al. 1997; Tenaillon et al. 2016; Good et al. 2017). These mutator populations gained

235 fitness slightly faster than the non-mutator populations (Wiser et al. 2013; Lenski et al. 2015).

236 However, genomic evolution in these populations was dominated by the accumulation of random

237 mutations (Tenaillon et al. 2016; Couce et al. 2017; Maddamsetti et al. 2017). For these reasons,

238 we made and tested separate predictions for the mutator and non-mutator populations. For the non-

239 mutator populations, where previous studies found compelling evidence for positive selection

240 (Woods et al. 2006; Tenaillon et al. 2016; Good et al. 2017), we predicted that aerobic-specific

241 genes would have more mutations than anaerobic-specific genes. By contrast, in the mutator

242 populations, previous studies indicated that random mutations (neutral or nearly neutral)

243 accumulated in those genes under relaxed selection, given the high mutation pressure. Of course,

244 some sites even within aerobic-specific genes could have accumulated neutral or nearly neutral

245 mutations in the oxic LTEE environment. However, purifying selection should lead to fewer 246 mutations in aerobic- than in anaerobic-specific genes in the mutator populations. 
To test these predictions, we examined the sets of 345 and 227 anaerobic and aerobic-

248 specific genes, respectively, as described in the Materials and Methods (supplemental file 1).

249 Given 4,143 protein-coding genes in the genome of the LTEE ancestor (Jeong et al. 2009), the

250 anaerobic- and aerobic-specific genes constitute $8.3 \%$ and $5.5 \%$ of that total, respectively. We

251 asked whether these two sets accumulated different numbers of mutations in clones sampled from

252 the LTEE populations at 50,000 generations. We controlled for differences in mutational target

253 size by summing over the length of the genes in each set. In clones from the six non-mutator

254 lineages, the aerobic-specific genes had 38 mutations, whereas the anaerobic-specific genes had

25521 mutations (two-tailed binomial test: $p<10^{-6}$ ). By contrast, the hypermutator clones had 836

256 mutations in anaerobic-specific genes and 333 mutations in aerobic-specific genes (two-tailed

257 binomial test: $p=0.0040$ ). The first result is consistent with stronger positive selection for

258 beneficial mutations in aerobic- than anaerobic-specific genes. The second result is consistent with

259 relaxed selection on anaerobic-specific genes, which could also be described as stronger purifying

260 selection on aerobic-specific genes.

261 Next, we examined these dynamics using whole-population metagenomic data that

262 includes mutations that fixed as well as those that reached a frequency above $\sim 5 \%$ in a population

263 through $\sim 60,000$ generations (Good et al. 2017). Rather than reanalyzing these data from scratch,

264 we analyzed the dataset previously generated by Good et al. (2017). We first visualized the

265 evolutionary dynamics for all mutations in aerobic- and anaerobic-specific genes in each

266 population (supplemental PDF, fig. S1). For the non-mutator populations (panels A-F in fig. S1),

267 mutations in aerobic-specific genes were more common than those in anaerobic-specific genes,

268 especially during the first 10,000 generations, indicating that many mutations in aerobic-specific

269 genes experienced positive selection. In the populations that evolved hypermutator phenotypes 
270 (panels G-L in fig. S1), however, it is difficult to tell by eye whether the rate of molecular

271 evolution differs between these two sets of genes. Therefore, we counted the number of observed

272 mutations in aerobic and anaerobic-specific genes in each population over time, normalized by

273 gene length.

When we examine the occurrence of nonsynonymous mutations, all six populations that

275 were never mutators, along with two others (Ara-1, Ara-3) before they became hypermutable,

276 have substantially more mutations in aerobic-specific genes than expected under the null

277 distribution calculated by resampling random sets of 227 genes (the cardinality of the aerobic-

278 specific genes) (fig. 2). The probability of this directional outcome occurring by chance under a

279 one-tailed binomial expectation is $(1 / 2)^{8}=1 / 256 \approx 0.004$, which is thus very significant. The

280 number of nonsynonymous mutations in anaerobic-specific genes in those same populations, by

281 contrast, is much lower in every case. For the mutator populations, including the two (Ara-1,

282 Ara-3) that evolved hypermutability fairly late in the LTEE, the rates of mutations in anaerobic-

283 and aerobic-specific genes track one another more closely. In at least three of these populations

284 (Ara-2, Ara+3, Ara+6), the rates of mutation accumulation decreased later in the LTEE. These

285 decelerations correspond to reversions or compensatory alleles that arose in those populations and

286 caused their mutation rates to decline (Tenaillon et al. 2016; Good et al. 2017). Some of these

287 populations also suggest a slower rate of mutation accumulation in aerobic- than in anaerobic-

288 specific genes, in particular near the end of the time course. Indeed, two mutator populations,

289 Ara+3 and Ara+6, accumulated significantly fewer nonsynonymous mutations in aerobic genes

290 than expected under the null distribution (non-parametric bootstrap with 10,000 replicates, $p<$

2910.0001 for each). The slower mutation accumulation in aerobic-specific genes suggests purifying

292 selection, as expected because the mutator phenotype increases the rate of deleterious mutations. 
293 It might also reflect, in part, saturation of possible beneficial mutations in aerobic-specific genes,

294 in accord with a "coupon-collecting" model of molecular evolution (Good et al. 2017). Population

295 Ara-4 is an outlier, however, in that its rate of mutation accumulation in aerobic-specific genes

296 slightly exceeded the rate observed in anaerobic-specific genes for most of its history, despite its

297 mutator phenotype (fig. 2).

298 To look more deeply into the role of purifying selection, we examined the accumulation of

299 insertions and deletions (indels), structural variants (including those generated by transposable

300 elements), and nonsense mutations in protein-coding genes in all 12 populations. These types of

301 mutations typically destroy protein function. Although such knockout mutations are sometimes

302 beneficial in evolution experiments (e.g., Cooper et al. 2001b), they would be highly deleterious

303 in conserved genes under purifying selection as well as in genes under positive selection to fine-

304 tune protein function (Maddamsetti et al. 2017). If aerobic-specific genes faced strong purifying

305 selection in the mutator populations, we reasoned that indels, structural variants, and nonsense

306 mutations would be underrepresented in them (fig. 3). Indeed, that was the case in four of the six

307 hypermutator populations (nonparametric bootstrap with 10,000 replicates: $p=0.0003$ for Ara-3;

$308 p=0.0014$ for Ara $-4 ; p<0.0001$ for Ara $+3 ; p=0.0003$ for Ara +6$)$. On balance, these observations

309 indicate stronger purifying selection on aerobic- than on anaerobic-specific genes in the LTEE,

310 especially in the populations that evolved hypermutable phenotypes. This finding is consistent

311 with the later evolution of anti-mutator alleles that reduced or reverted mutation rates to the

312 ancestral level in most of the populations that evolved hypermutability. The fact that mutations

313 accumulated more slowly in anaerobic-specific genes than expected under the null distribution in

314 two mutator populations (nonparametric bootstrap with 10,000 replicates: $p<0.0001$ for Ara +3 ; 
$315 p=0.0035$ for Ara+6) suggests that some anaerobic-specific genes might also have experienced

316 purifying selection, indicating functionality even during aerobic growth.

317 For synonymous mutations, which are effectively neutral in the vast majority of cases, we

318 expect to see many more of them in the mutator populations, and indeed that is the case. We also

319 do not expect to see any systematic association with aerobic- or anaerobic-specific genes. That

320 expectation is also fulfilled: three of the six mutator populations had more synonymous mutations

321 in aerobic- than in anaerobic-specific genes, and the other three show the opposite trend

322 (supplemental PDF, fig. S2). It is a bit puzzling that the difference between the two gene sets is so

323 noticeable in some cases in one direction or the other. These differences might reflect hitchhiking,

324 whereby several synonymous mutations affecting one or the other gene set, all on the same

325 background, were pushed to high frequency (or pulled to extinction) in a particular population

326 (Maddamsetti et al. 2015). In any case, there is no overall pattern across the six mutator populations

327 for synonymous mutations (supplemental PDF, fig. S2).

\section{Mutations in Genes that Regulate Metabolic Plasticity in Response to Oxygen}

331 After establishing the genome-wide signatures of selection on aerobic- and anaerobic-specific

332 genes, we now turn our attention to three particular genes known to regulate metabolic plasticity

333 in response to oxygen availability: $f n r, \operatorname{arc} A$, and $\operatorname{arcB}$ (fig. 1). Only two LTEE populations, both

334 mutators (Ara+3, Ara+6), have nonsynonymous mutations in the fnr gene; in both populations, the

335 mutations arose well after the populations had evolved hypermutability. By contrast, 11 of the 12

336 populations have nonsynonymous mutations in $\operatorname{arc} A, \operatorname{arcB}$, or both (fig. 4A). The other population

337 (Ara-6) has a 9-bp deletion in arcA. In another population, Ara-2, two lineages designated $\mathrm{S}$ and 
L have coexisted since about generation 6,000 (Rozen et al. 2005), and only the S lineage has a mutation in $\operatorname{arc} A$. Many of the mutations in $\operatorname{arc} A$ and $\operatorname{arc} B$ were already present in the clones sequenced at 10,000 generations (fig. 4A), and $\operatorname{arc} A$ was previously identified as showing a

341 signature of strong positive selection in the LTEE (Tenaillon et al. 2016). We mapped the $\operatorname{arcA}$

342 and $\operatorname{arcB}$ mutations in the 50,000-generation clones onto the encoded protein structures. Mutations

343 in $\operatorname{arc} A$ impact both the response regulator and DNA binding domains of the protein (fig. 4B), and

344 mutations in $\operatorname{arcB}$ map to several protein domains including the histidine kinase and histidine

345 kinase receptor (fig. 4C). There are several ways that these mutations might affect metabolic 346 plasticity in the evolved bacteria. Mutations in either gene could affect the stability of the proteins

347 or their activities, thereby (i) altering the capacity of ArcB to sense redox changes through the

348 quinone pool; (ii) altering the rate or efficiency of phosphoryl transfer between the ArcB protein

349 domains; (iii) decreasing the extent of ArcA phosphorylation; (iv) increasing the rate of ArcA

350 dephosphorylation; (v) decreasing the extent of ArcA phosphorylation-dependent oligomerization;

351 or (vi) altering the DNA binding efficiency of ArcA. In any case, these mutations may impact

352 Arc AB signaling and might thus affect the ability of the evolved strains to grow in an anoxic

353 environment.

Fitness of Evolved Bacteria Under Oxic and Anoxic Conditions

357 Half of the LTEE populations were founded by E. coli B strain REL606 and half by REL607, an $358 \operatorname{araA}$ mutant of REL606. This mutation is selectively neutral in the LTEE environment (Lenski et 359 al. 1991; Wiser et al. 2013), and it provides a readily scored marker for distinguishing competitors 360 in assays of relative fitness. However, it was unknown whether the araA mutation is also neutral 
under anoxic conditions. To that end, we competed REL606 and REL607 in oxic and anoxic environments, with other conditions the same as those used in the LTEE. We saw no significant differences in relative fitness in either the oxic $(t=0.6972$, d.f. $=4$, two-tailed $p=0.5241)$ or anoxic $(t=1.1433$, d.f. $=4$, two-tailed $p=0.3167)$ environment. Thus, the araA mutation can serve as a useful marker for assaying the relative fitness of evolved and ancestral clones in both the anoxic and oxic environments.

We expected that the evolved bacteria would be better adapted to the oxic environment, where they evolved, than to the anoxic environment. To test this hypothesis, we competed clones sampled at 2,000, 10,000, and 50,000 generations against the reciprocally marked ancestral strains. As explained in the Materials and Methods section, we excluded one population (Ara+6) at all

371 three time points, and two others (Ara-2, Ara-3) at the last time point, because of technical 372 difficulties associated with enumerating these competitors. We calculated fitness as the ratio of the 373 realized growth rate of the evolved clone relative to that of the ancestor during a competition. This 374 metric integrates the effects of differences in lag, growth, and stationary phases (Lenski et al. 1991; 375 Vasi et al. 1994). Figure 5 shows the results obtained for each evolved clone, with standard errors 376 based on replicate competition assays for that clone. Note that all points lie below the isocline 377 corresponding to equal fitness in the anoxic and oxic environments, consistent with our hypothesis.

378 As an overall assessment, we performed paired comparisons of fitness in the two environments at 379 each time point, and in all cases the difference was highly significant (paired $t$-tests; generation $3802,000: t=8.7849, \mathrm{df}=10$, one-tailed $p<0.0001$; generation 10,000: $t=8.7986, \mathrm{df}=10$, one-tailed $381 p<0.0001$; generation 50,000: $t=5.9556, \mathrm{df}=8$, one-tailed $p=0.0002)$. Indeed, all 31 clones 382 tested had higher estimated fitness values in the oxic environment than in the anoxic one (sign test, 
$p<<0.0001)$. Figure 6 shows the grand mean fitness values in the two environments over time,

384 with confidence limits based on the replicate populations. throughout the experiment (Lenski et al. 2015), and our data recapitulate this behavior (fig. 6,

387 supplemental PDF, fig. S3). Although we expected, and confirmed (figs. 5, 6), that fitness relative

388 to the ancestor would be higher in the oxic environment than in the anoxic environment, we did not have a clear expectation for the trajectory of fitness relative to the ancestor in the novel anoxic environment. On the one hand, the anoxic environment shares most aspects of the oxic

predators. On the other hand, some aspects of performance might tradeoff between the two

by mutation accumulation, especially in the mutator populations. Given these opposing expectations, one might expect anaerobic fitness to follow a quasi-random walk (Freckleton and Harvey 2006). The fitness trajectories measured in the anoxic environment show some apparent changes in direction, but the differences between consecutive time points are generally within the margin of error (supplemental PDF, fig. S3). In fact, none of the 31 comparisons between sequential points are significant at $p<0.05$ after performing a Bonferroni correction. In any case,

400 fitness tended to increase even in the anoxic environment in most populations (supplemental PDF, 401 fig. S3). Across all of the clones tested, 23 of 31 had point estimates of their fitness relative to the 402 ancestor in the anoxic environment greater than unity (two-tailed sign test, $p=0.0107$ ). However, 403 the grand mean fitness of the evolved bacteria in that environment was significantly greater than 404 unity only at the 10,000-generation time point (fig. 6). 
408 Each population acquired a unique set of mutations over the course of the LTEE. However, 409 adaptation to the common environment contributed to strong parallelism at the level of genes, 410 especially in the non-mutator populations (Woods et al. 2006; Tenaillon et al. 2016; Good et al. 411 2017). For example, just 57 genes that make up only $\sim 2 \%$ of the coding genome had $\sim 50 \%$ of the 412 nonsynonymous mutations that accumulated in the non-mutator populations through 50,000 413 generations (Tenaillon et al. 2016). The trajectories for fitness also showed strong parallelism 414 (Lenski and Travisano 1994; Wiser et al. 2013; Lenski et al. 2015). For example, the square root 415 of the among-population variance for fitness was only 5\% after 50,000 generations (Lenski et al. 416 2015), when the grand mean fitness itself had increased by $70 \%$ (Wiser et al. 2013). By contrast, 417 with relaxed selection on anaerobic-specific genes, the accumulation of different sets of mutations 418 should contribute to the populations having greater heterogeneity in fitness when assayed in the 419 anoxic environment than in the oxic environment. We first examined these predictions by 420 performing six one-way ANOVAs (two environments and three time points) to test whether the 421 among-lineage variation was significant (table 1). At 2,000 generations, we saw significant fitness 422 heterogeneity in the anoxic environment, consistent with our expectation. At 10,000 generations, 423 by contrast, there was no significant heterogeneity in either environment. Finally, we saw 424 significant fitness variation in both test environments after 50,000 generations.

425 Of course, statistical significance, or the lack thereof, is a crude criterion by which to 426 compare the among-lineage heterogeneity in fitness between the two environments. One can 427 visualize the magnitude of the heterogeneity by estimating the variance attributable to lineages 428 that is greater than expected from the measurement error across replicate assays. However, the 
statistical uncertainty in estimating variance components is often quite large, and indeed that was the case in our analyses, which showed no significant difference in fitness heterogeneity between

431 the oxic and anoxic environments at any of the generations tested (fig. 7). We also considered the

432 possibility that these analyses were unduly influenced by the subset of populations that evolved

433 hypermutability, which might obscure differences in the among-lineage variation between the two

434 environments. To that end, we repeated the ANOVAs (supplemental PDF, table S2) and the

435 estimation of variance components using only those lineages that did not evolve hypermutability.

436 However, the results of these analyses were not appreciably different (supplemental PDF, fig. S5).

437 In short, contrary to our expectation, we found no compelling evidence of greater among-lineage

438 heterogeneity when fitness was measured in the anoxic than in the oxic environment.

\section{Discussion}

442 If a trait or function is no longer useful to an organism because of a change in its environment,

443 then it may be lost over time. It is unclear, however, what factors determine whether and how

444 quickly traits under relaxed selection will be lost. In this study, we investigated the consequences

445 of relaxed selection for metabolic plasticity by examining the maintenance of anaerobic

446 metabolism - an ancient and core function - using E. coli strains that have been evolving in the

447 laboratory under strictly oxic conditions for more than 70,000 generations. On the one hand, one

448 can really imagine that anaerobic metabolism would decay because it has been unused during that

449 time. On the other hand, one can imagine that anaerobic metabolism would be maintained even

450 under relaxed selection owing to the ancient, tightly intertwined physiological and genetic

451 networks that govern aerobic and anaerobic metabolism. 
Two mechanisms have been proposed to explain trait loss under relaxed selection (Fong et

453 al. 1995; Cooper and Lenski 2000): antagonistic pleiotropy (AP), and mutational degradation

454 (MD). AP is a selection-driven process whereby improvements to traits that are beneficial in one

455 environment trade off with traits that are useful in other conditions (Williams 1957). An example

456 of AP seen in the LTEE is the loss of the ability to grow on maltose, which has occurred in most

457 populations by mutations in a gene that encodes a transcriptional activator of other genes that

458 encode proteins used to transport and metabolize that sugar (Pelosi et al. 2006; Leiby and Marx

459 2014). In the absence of maltose, the loss of expression of those genes confers a demonstrable

460 competitive advantage, indicative of AP. A familiar example in the realm of multicellular

461 eukaryotes is senescence, whereby traits that increase reproductive potential early in life reduce

462 survival late in life (Rodríguez et al. 2017). By contrast, MD occurs by a neutral process. In this

463 scenario, traits under relaxed selection accumulate degradative mutations that are neutral in an

464 organism's current environment, but which reduce the organism's fitness in environments where

465 those traits are useful. AP and MD are not mutually exclusive, so both can act together to degrade

466 unused functions. In those LTEE populations that evolved greatly elevated mutation rates, one

467 would expect MD to cause much greater decay of unused genes (Cooper and Lenski 2000; Couce

468 et al. 2017). The hypermutable lineages have also increased their fitness in the LTEE environment

469 more than the populations that retained the low ancestral mutation rate, although the difference is

470 small (Wiser et al. 2013; Lenski et al. 2015). Whether the LTEE populations would, on balance,

471 lose fitness under anoxic conditions, and whether those lineages that evolved hypermutability

472 would lose fitness to a greater extent, depends on the form and strength of the regulatory and

473 physiological couplings between aerobic and anaerobic metabolism. 
We examined genomic and metagenomic sequence data to detect possible signatures of different modes of selection acting on aerobic- and anaerobic-specific genes. Six of the 12 LTEE

476 populations retained the low ancestral point-mutation rate throughout their history (Tenaillon et

477 al. 2016; Good et al. 2017). All six accumulated many more nonsynonymous mutations in aerobic-

478 specific genes than in anaerobic-specific genes (fig. 2). Moreover, these mutations were

479 concentrated in a subset of genes, with that genetic parallelism indicative of adaptive evolution

480 (Tenaillon et al. 2016; Good et al. 2017). In a number of cases, the inferred benefit of parallel

481 changes was confirmed by measuring the relative fitness of otherwise isogenic strains (Barrick et

482 al. 2009). By contrast, the six populations that evolved hypermutability (Sniegowski et al. 1997;

483 Wielgoss et al. 2013; Tenaillon et al. 2016) accumulated more nonsynonymous mutations in

484 anaerobic- than in aerobic-specific genes (fig. 2). Consistent with purifying selection against

485 mutations in aerobic-specific genes, presumptive knockout mutations (insertions, deletions,

486 nonsense mutations, and structural variants) were underrepresented in those genes in most of the

487 hypermutable populations (fig. 3). More generally, our results agree with several studies that have

488 found a higher frequency of mutations in genes underlying traits under relaxed selection (Shabalina

489 et al. 1997; Cooper and Lenski 2000; Funchain et al. 2000; Maughan et al. 2007; Shewaramani et

490 al. 2017; Cui et al. 2019; Harrison et al. 2019). For example, Maughan et al. (2007) propagated

491 Bacillus subtilis vegetatively (i.e., without sporulation) for 6,000 generations. They found that the

492 evolved strains' inability to generate spores was driven largely by the accumulation of mutations

493 in genes required for sporulation, but not for vegetative growth.

We also identified mutations in $\operatorname{arc} A$ and $\operatorname{arc} B$ in almost all of the evolved lines (fig. 4).

495 These genes encode the two-component system responsible for regulating the switch between 496 aerobic and anaerobic metabolism (fig. 1). Previous work found that $\operatorname{arc} A$ is among the top 15 
genes in the LTEE in terms of nonsynonymous substitutions among non-hypermutable lineages, and this parallelism implies positive selection (Tenaillon et al. 2016). Moreover, $\operatorname{arcB}$ mutations have been implicated in improving growth on acetate - a waste product of glucose metabolismin some LTEE populations (Plucain et al. 2014; Quandt et al. 2015; Leon et al. 2018). populations (fig. 2), along with numerous mutations in $\operatorname{arc} A B$ in both mutator and non-mutator populations (fig. 4), one might reasonably expect that anaerobic metabolism would be degraded,

504 or perhaps even completely lost, in the LTEE populations. However, that was not the case. Our 505 results indicate a more nuanced outcome. On average, the grand mean fitness measured under anoxic conditions tended to increase over the first 10,000 generations of the LTEE, although to a much lesser extent than fitness measured under oxic conditions (fig. 6). Between 10,000 and 50,000 generations, the grand mean fitness measured in the anoxic environment showed no clear trend, even as fitness under the oxic conditions of the LTEE continued to increase, albeit at a

510 slower rate (fig. 6). One might also expect to see greater variation in fitness among the replicate 511 lines when measured in the novel anoxic environment than in the oxic environment where selection 512 was in force during the LTEE. Substantially increased among-population variation in fitness has 513 been reported, for example, when growth substrates (Travisano and Lenski 1996) and temperature 514 were changed (Cooper et al. 2001a). However, we found no meaningful differences in the among515 population variance in fitness at any of the time points tested (fig. 7). We also saw no consistent 516 difference in fitness in the anoxic environment between the hypermutable and non-mutator 517 populations (supplemental PDF, fig. S4), despite the compelling evidence for relaxed selection on 518 anaerobic-specific genes in the hypermutable populations (fig. 2). These results broadly suggest 519 that anaerobic metabolism, taken as a whole, did not decay appreciably under relaxed selection, 
520 perhaps owing to conserved underlying correlations with traits that contribute to aerobic

521 performance and that experienced a mixture of positive and purifying selection during the LTEE.

522 Similar correlated responses have been measured for other phenotypic traits in the LTEE

523 populations. For example, the fitness gains made on glucose during the first 2,000 generations led

524 to correlated improvements on lactose (Travisano and Lenski 1996). Leiby and Marx (2014)

525 measured the growth of clones isolated at generations 20,000 and 50,000 on a large array of

526 substrates. They found correlated improvements on some substrates that the populations had not

527 seen for decades, including a few that the ancestors could not even use. Cooper (2002) competed

528 evolved and ancestral strains in four different media, including two with altered glucose

529 concentrations (DM2.5 and DM250), the base medium (DM25) with added bile salts, and a dilute

530 version of the nutritionally complex LB medium. The relative fitness of the evolved lines had, on

531 average, increased in all of these foreign environments. In another study, Meyer et al. (2010)

532 showed that most late-generation LTEE lines had evolved resistance to phage lambda, despite

533 never being exposed to lambda or any other phage during the experiment. They further showed

534 that this positive correlated response was itself associated with a negative correlated response,

535 namely the loss of the capacity to grow on maltose. Lambda uses a maltose transporter to infect

536 cells, and reduced expression of that protein promotes fitness on glucose, compromises growth on

537 maltose, and confers resistance to lambda (Meyer et al. 2010).

538 What might contribute to the maintenance and even slight improvement of anaerobic

539 performance, despite relaxed selection? The evolved populations showed modest improvement,

540 on average, in the anoxic environment at 10,000 generations (fig. 6). One possibility is that some

541 traits and pathways, such as faster glucose transport and glycolysis, are beneficial in both oxic and

542 anoxic environments. As a consequence, some of the mutated genes that evolved in parallel early 
543 in the LTEE might be beneficial in the anoxic environment as well. Only 4 of the 15 genes $(p y k F$,

$544 h s l U$, infB, and $r p l F$ ) with the strongest signatures of parallel evolution (Tenaillon et al. 2016) are

545 in the aerobic-specific gene set, and even some of them might sometimes contribute to anaerobic

546 metabolism. This overlap may explain the correlated improvement in anaerobic fitness early in the

547 LTEE. However, it is unclear whether it is sufficient to explain the maintenance of anaerobic

548 growth capacity after 50,000 generations of relaxed selection, especially in those lines that were

549 hypermutable for much of that time, because knocking out even a single function that is truly

550 required for anaerobic growth should disrupt that metabolic plasticity. In any case, future work

551 might examine the fitness effects of specific mutations that arose in the LTEE, including those that

552 fixed early in the LTEE, on anaerobic fitness to determine if they do, in fact, provide correlated

553 advantages.

Another possible explanation for the maintenance of anaerobic performance hinges on a

555 connection between biochemistry and metabolism. The formulation of the DM25 medium does

556 not provide an alternative terminal electron acceptor that would permit anaerobic respiration. Thus,

557 the bacteria are presumably fermenting glucose in the anoxic environment. Even in the oxic

558 environment of the LTEE, however, the cells might be fermenting rather than respiring glucose,

559 using a process called overflow metabolism (Basan et al. 2015; Swain and Fagan 2019). The use

560 of overflow metabolism would favor the maintenance and even improvement of genes involved in

561 fermentative metabolism. Acetate is a major byproduct of fermentative metabolism, and the fact

562 that several LTEE populations evolved frequency-dependent interactions mediated by cross-

563 feeding on acetate (Elena and Lenski 1997; Rozen et al. 2005; Großkopf et al. 2016; Leon et al.

564 2018) provides support for this hypothesis. A prediction of this hypothesis is that the bacteria in

565 the LTEE are not respiring, and therefore not using dissolved oxygen, at least during some phase 
566 of their population growth. Future studies can test this prediction, and they could also use

567 alternative terminal electron acceptors to measure the respiratory capacity of the evolved lines

568 under anoxic conditions to determine whether their anaerobic growth has become restricted to

569 fermentative metabolism.

570 A third explanation for the maintenance and even slight improvement in anaerobic fitness

571 of the LTEE populations involves the ArcAB system (fig. 1). These two proteins work in concert

572 to regulate the expression of genes relevant to both aerobic and anaerobic growth (Iuchi and Lin

573 1988; Gunsalus and Park 1994; Unden and Bongaerts 1997). In particular, ArcA and ArcB together

574 repress the genes that encode TCA-cycle enzymes under anoxic conditions. In their seminal paper

575 describing this system, Iuchi and Lin (1988) isolated $\operatorname{arc} A$ mutants that produce abnormally high

576 levels of enzymes that are normally repressed under anoxic conditions. These mutants were

577 pleiotropic, so that several aerobic-specific enzymes had increased expression under anoxic

578 conditions including those involved in the TCA cycle and in fatty acid degradation as well as some

579 flavoprotein dehydrogenases and a ubiquinone oxidase. Saxer et al. (2014) also reported extensive

580 metabolic changes caused by $\operatorname{arc} A$ mutations in short-term evolution experiments with both $E$. coli

581 and Citrobacter freundii. They performed proteomic analyses that recapitulated the changes in

582 gene expression reported by Iuchi and Lin (1988), and they also saw increased expression of genes

583 involved in amino-acid metabolism. Saxer et al. (2014) concluded that mutations in global

584 regulators like the $\mathrm{ArcAB}$ system could, in one step, expand the niche of an organism by

585 substantially remodeling its cellular metabolism.

All of these potential physiological and genetic explanations for the unexpectedly strong

587 performance of the evolved LTEE bacteria when tested under anoxic conditions might fit under a

588 common theme, which has been called "buttressing pleiotropy" (Lahti et al. 2009). In contrast to 
the tradeoffs (negative correlations) generated by antagonistic pleiotropy, the idea of buttressing pleiotropy is that "the function of the correlated trait is buttressing or maintaining values of the

591 focal trait" (Lahti et al. 2009). The relevance of buttressing pleiotropy to our results would be

592 strengthened if the genetic architecture underlying aerobic and anaerobic metabolism had many

593 connections. To that end, we used empirical and computationally derived information on protein-

594 protein interactions to infer and visualize the topology of the aerobic- and anaerobic-specific gene

595 sets in our study (Shannon et al. 2003; Szklarczyk et al. 2017). One immediately sees many

596 connections that demonstrate these two sets are far from independent (fig. 8). It is quite possible,

597 therefore, that selection for improved aerobic growth would buttress anaerobic performance.

A related idea, also relevant to our system, is robustness. E. coli is often described as a

599 facultative anaerobe because it can grow not only in the well-oxygenated conditions widely used

600 in most laboratories, but also when deprived of oxygen in special growth chambers. However, $E$.

601 coli might be better described as a facultative aerobe, because its natural home is the anoxic

602 mammalian colon. To be sure, many E. coli cells periodically exit their hosts, and the ability to

603 survive in the presence of oxygen is essential for colonizing new hosts. However, we would

604 suggest that most of the 100-million year or so history of this species has been spent living under

605 anerobic conditions - even if there are as many E. coli cells outside as inside mammalian hosts at

606 any given time, those that are outside are much more likely to be evolutionary dead-ends. If so,

607 then evolution might have favored a more robust anaerobic metabolism, one that could not easily

608 be disrupted by short-sighted selection for improved fitness in the oxic environment. A test of this

609 anaerobic-robustness hypothesis would be to perform an experiment identical to the LTEE, except

610 in a strictly anoxic environment. If populations that evolved for 50,000 generations in the absence 
611 of oxygen lost the ability to grow in its presence, then that would indicate that aerobic metabolism

612 is less robust that anaerobic metabolism.

613 In a different vein, it is known that some enzymes exhibit substrate promiscuity (Nam et

614 al. 2012). Changes to global regulators - including the ArcAB system and others that have changed

615 during the LTEE (Cooper et al. 2003, 2008; Philippe et al. 2007; Crozat et al. 2011)—might

616 increase expression of some promiscuous enzymes, and those latent functions might yield benefits

617 in multiple environments. Future work might compare the transcriptomes of ancestral and evolved

618 bacteria in oxic and anoxic environments, as well as examine the effects of specific mutations to

619 ArcAB and other global regulators on the transcriptomes. Another area for future work would

620 examine the speed with which the ancestral and evolved lines can respond to sudden changes in

621 the environment from oxic to anoxic and vice versa. Yet another interesting direction might use a

622 combination of genetic engineering and experimental evolution to generate obligate aerobic and

623 obligate anaerobic strains, which could then be studied to better understand the constraints on each

624 type of metabolism.

625 All in all, our results show that anaerobic metabolism was surprisingly robust during

62650,000 generations of adaptation to a strictly oxic environment. The capacity for anaerobic growth

627 persisted even in those lineages that evolved hypermutability, despite genetic signatures that

628 showed increased mutation accumulation in anaerobic-specific genes in those lines. Thus, relaxed

629 selection on a functional trait does not always result in its loss. We suggest that one must know

630 how the genes and proteins responsible for traits that experience relaxed selection interact with the

631 rest of an organism's genome and physiology, in order to understand the potential for loss,

632 maintenance, or even correlated improvement of such traits. More generally, investigating the role

633 of genetic architecture in the evolutionary process might help us better predict how organisms will 
634 respond to novel selection pressures and environmental perturbations, including climate change.

635 Such understanding may also suggest new ways of promoting or constraining the evolutionary

636 trajectories of organisms and their traits, which could be used for synthetic biology, on the one

637 hand, and to limit the evolution of pathogens, on the other hand.

\section{Acknowledgments}

641 We thank Terence Marsh, Charles Ofria, Gemma Reguera, and Chris Waters for feedback as this

642 research progressed; Zachary Blount for helpful comments on the manuscript; and members of the

643 Lenski lab for valuable discussions. We thank Terence Marsh for providing access to an anaerobic

644 chamber, and the MSU Department of Microbiology and Molecular Genetics for related supplies.

645 This work was supported in part by a grant from the National Science Foundation (currently DEB-

646 1951307), the BEACON Center for the Study of Evolution in Action (DBI-0939454), and the

647 USDA National Institute of Food and Agriculture (MICL02253). Any opinions, findings, and

648 conclusions or recommendations expressed in this material are those of the authors and do not

649 necessarily reflect the views of the funders. 


\section{Literature Cited}

652

653

654

655

656

657

658

659

660

661

662

663

664

665

666

667

668

Altenhoff, A. M., N. M. Glover, C. M. Train, K. Kaleb, A. Warwick Vesztrocy, D. Dylus, T. M. De Farias, et al. 2018. The OMA orthology database in 2018: Retrieving evolutionary relationships among all domains of life through richer web and programmatic interfaces. Nucleic Acids Research 46(D1):D477-D485.

Barrick, J. E., D. Yu, S. Yoon, H. Jeong, T. Oh, D. Schneider, R. E. Lenski, et al. 2009. Genome evolution and adaptation in a long-term experiment with Escherichia coli. Nature 461:12431247.

Basan, M., S. Hui, H. Okano, Z. Zhang, Y. Shen, J. R. Williamson, and T. Hwa. 2015. Overflow metabolism in Escherichia coli results from efficient proteome allocation. Nature 528:99-104.

Bennett, A. F., K. M. Dao, and R. E. Lenski. 1990. Rapid evolution in response to high-temperature selection. Nature 346:79-81.

Blazejewski, T., H. I. Ho, and H. H. Wang. 2019. Synthetic sequence entanglement augments stability and containment of genetic information in cells. Science 365:595-598.

Cooper, T. F., D. E. Rozen, and R. E. Lenski. 2003. Parallel changes in gene expression after 20,000 generations of evolution in Escherichia coli. Proceedings of the National Academy of Sciences of the USA 100:1072-1077.

Cooper, T. F., S. K. Remold, R. E. Lenski, and D. Schneider. 2008. Expression profiles reveal parallel evolution of epistatic interactions involving the CRP regulon in Escherichia coli. PLoS Genetics 4:e35.

Cooper, V. S. 2002. Long-term experimental evolution in Escherichia coli. X. Quantifying the fundamental and realized niche. BMC Evolutionary Biology 2:12.

Cooper, V. S., and R. E. Lenski. 2000. The population genetics of ecological specialization in evolving Escherichia coli populations. Nature 407:736-739.

Cooper, V. S., A. F. Bennett, and R. E. Lenski. 2001a. Evolution of thermal dependence of growth rate of Escherichia coli populations during 20,000 generations in a constant environment. Evolution 55:889-896.

Cooper, V. S., D. Schneider, M. Blot, and R. E. Lenski. 2001b. Mechanisms causing rapid and parallel losses of ribose catabolism in evolving populations of Escherichia coli B. Journal of Bacteriology 183:2834-2841.

Couce, A., L. V. Caudwell, C. Feinauer, T. Hindré, J. P. Feugeas, M. Weigt, R. E. Lenski, et al. 2017. Mutator genomes decay, despite sustained fitness gains, in a long-term experiment with bacteria. Proceedings of the National Academy of Sciences of the USA 114:E9026-E9035.

Crozat, E., T. Hindré, L. Kühn, J. Garin, R. E. Lenski, and D. Schneider. 2011. Altered regulation of the OmpF porin by Fis in Escherichia coli during an evolution experiment and between B and K-12 strains. Journal of Bacteriology 193:429-440.

Cui, R., T. Medeiros, D. Willemsen, L. N. M. Iasi, G. E. Collier, M. Graef, M. Reichard, et al. 2019. Relaxed selection limits lifespan by increasing mutation load. Cell 178:385-399.

Darwin, C. 1859. On the Origin of Species. John Murray, London.

Elena, S. F., and R. E. Lenski. 1997. Long-term experimental evolution in Escherichia coli. VII. 
mechanisms maintaining genetic variability within populations. Evolution 51:1058-1067.

Elena, S. F., and R. E. Lenski. 2003. Evolution experiments with microorganisms: the dynamics and genetic bases of adaptation. Nature Reviews Genetics 4:457-469.

Fong, D. W., T. C. Kane, and D. C. Culver. 1995. Vestigialization and loss of nonfunctional characters. Annual Review of Ecology and Systematics 26:249-268.

Frankel, N., G. K. Davis, D. Vargas, S. Wang, F. Payre, and D. L. Stern. 2010. Phenotypic robustness conferred by apparently redundant transcriptional enhancers. Nature 466:490-493.

Fraser, H. B., and E. E. Schadt. 2010. The quantitative genetics of phenotypic robustness. PLoS ONE 5:e8635.

Freckleton, R. P., and P. H. Harvey. 2006. Detecting non-Brownian trait evolution in adaptive

Funchain, P., A. Yeung, J. L. Stewart, R. Lin, M. M. Slupska, and J. H. Miller. 2000. The consequences of growth of a mutator strain of Escherichia coli as measured by loss of function among multiple gene targets and loss of fitness. Genetics 154:959-970.

Geng, P., S. P. Leonard, D. M. Mishler, and J. E. Barrick. 2019. Synthetic genome defenses against selfish DNA elements stabilize engineered bacteria against evolutionary failure. ACS Synthetic Biology 8:521-531.

Good, B. H., M. J. McDonald, J. E. Barrick, R. E. Lenski, and M. M. Desai. 2017. The dynamics of molecular evolution over 60,000 generations. Nature 551:45-50.

Gordon, D. M., and A. Cowling. 2003. The distribution and genetic structure of Escherichia coli in Australian vertebrates: Host and geographic effects. Microbiology 149:3575-3586.

Großkopf, T., J. Consuegra, J. Gaffé, J. C. Willison, R. E. Lenski, O. S. Soyer, and D. Schneider. 2016. Metabolic modelling in a dynamic evolutionary framework predicts adaptive diversification of bacteria in a long-term evolution experiment. BMC Evolutionary Biology 16:163.

Gunsalus, R. P., and S. J. Park. 1994. Aerobic-anaerobic gene regulation in Escherichia coli: Control by the ArcAB and Fnr regulons. Research in Microbiology 145:437-450.

Harrison, M. C., E. B. Mallon, D. Twell, and R. L. Hammond. 2019. Deleterious mutation accumulation in Arabidopsis thaliana pollen genes: A role for a recent relaxation of selection. Genome Biology and Evolution 11:1939-1951.

Iuchi, S., and E. C. Lin. 1988. arcA (dye), a global regulatory gene in Escherichia coli mediating repression of enzymes in aerobic pathways. Proceedings of the National Academy of Sciences of the USA 85:1888-1892.

Jeong, H., V. Barbe, C. H. Lee, D. Vallenet, D. S. Yu, S.-H. Choi, A. Couloux, et al. 2009. Genome sequences of Escherichia coli B strains REL606 and BL21(DE3). Journal of Molecular Biology 394:644-652.

Jia, D., M. Lu, K. H. Jung, J. H. Park, L. Yu, J. N. Onuchic, B. A. Kaipparettu, et al. 2019. Elucidating cancer metabolic plasticity by coupling gene regulation with metabolic pathways. Proceedings of the National Academy of Sciences of the USA 116:3909-3918.

Kang, Y., K. D. Weber, Y. Qiu, P. J. Kiley, and F. R. Blattner. 2005. Genome-wide expression 
analysis indicates that FNR of Escherichia coli $\mathrm{K}-12$ regulates a large number of genes of unknown function. Journal of Bacteriology 187:1135-1160.

Keseler, I. M., A. Mackie, A. Santos-Zavaleta, R. Billington, C. Bonavides-Martínez, R. Caspi, C. Fulcher, et al. 2017. The EcoCyc database: Reflecting new knowledge about Escherichia coli K-12. Nucleic Acids Research 45:D543-D550.

Kwon, O., D. Georgellis, E.C. Lin. 2003. Rotational on-off switching of a hybrid membrane sensor kinase Tar-ArcB in Escherichia coli. Journal of Biological Chemistry 278:13192-13195.

Lahti, D. C., N. A. Johnson, B. C. Ajie, S. P. Otto, A. P. Hendry, D. T. Blumstein, R. G. Coss, et al. 2009. Relaxed selection in the wild. Trends in Ecology and Evolution 24:487-496.

Lamrabet, O., M. Martin, R. E. Lenski, and D. Schneider. 2019. Changes in intrinsic antibiotic susceptibility during a long-term evolution experiment with Escherichia coli. mBio 10:e018919.

Leiby, N., and C. J. Marx. 2014. Metabolic erosion primarily through mutation accumulation, and not tradeoffs, drives limited evolution of substrate specificity in Escherichia coli. PLoS Biology 12:e1001789.

Lenski, R. E. 2017. Experimental evolution and the dynamics of adaptation and genome evolution in microbial populations. ISME Journal 11:2181-2194.

Lenski, R. E., and M. Travisano. 1994. Dynamics of adaptation and diversification: a 10,000generation experiment with bacterial populations. Proceedings of the National Academy of Sciences of the USA 91:6808-6814.

Lenski, R. E., M. R. Rose, S. C. Simpson, and S. C. Tadler. 1991. Long-term experimental evolution in Escherichia coli. I. Adaptation and divergence during 2,000 generations. American Naturalist 138:1315-1341.

Lenski, R. E., C. Ofria, R. T. Pennock, and C. Adami. 2003. The evolutionary origin of complex features. Nature 423:139-144.

Lenski, R. E., J. E. Barrick, and C. Ofria. 2006. Balancing robustness and evolvability. PLoS Biology 4:e428.

Lenski, R. E., M. J. Wiser, N. Ribeck, Z. D. Blount, J. R. Nahum, J. J. Morris, L. Zaman, et al. 2015. Sustained fitness gains and variability in fitness trajectories in the long-term evolution experiment with Escherichia coli. Proceedings of the Royal Society B 282:20152292.

Leon, D., S. D'Alton, E. M. Quandt, and J. E. Barrick. 2018. Innovation in an E. coli evolution experiment is contingent on maintaining adaptive potential until competition subsides. PLoS Genetics 14:e1007348.

Maddamsetti, R., R. E. Lenski, and J. E. Barrick. 2015. Adaptation, clonal interference, and frequency-dependent inter
Genetics 200:619-631.

Maddamsetti, R., P. J. Hatcher, A. G. Green, B. L. Williams, D. S. Marks, and R. E. Lenski. 2017. Core genes evolve rapidly in the long-term evolution experiment with Escherichia coli. Genome Biology and Evolution 9:1072-1083.

Maughan, H., J. Masel, C. W. Birky, and W. L. Nicholson. 2007. The roles of mutation 
accumulation and selection in loss of sporulation in experimental populations of Bacillus subtilis. Genetics 177: 937-948.

Maughan, H., C. W. Birky, and W. L. Nicholson. 2009. Transcriptome divergence and the loss of plasticity in Bacillus subtilis after 6,000 generations of evolution under relaxed selection for sporulation. Journal of Bacteriology 191:428-433.

Melville, S. B., and R. P. Gunsalus. 1990. Mutations in fnr that alter anaerobic regulation of electron transport-associated genes in Escherichia coli. Journal of Biological Chemistry 265:18733-18736.

Meyer, J. R., A. A. Agrawal, R. T. Quick, D. T. Dobias, D. Schneider, and R. E. Lenski. 2010. Parallel changes in host resistance to viral infection during 45,000 generations of relaxed selection. Evolution 64:3024-3034.

Müller, H. E. 1977. Age and evolution of bacteria. Experientia 33:979-984.

Nam, H., N. E. Lewis, J. A. Lerman, D.-H. Lee, R. L. Chang, D. Kim, and B. O. Palsson. 2012. Network context and selection in the evolution to enzyme specificity. Science 337:1101-1104.

Nijhout, F. H., F. Sadre-Marandi, J. Best, and M. C. Reed. 2017. Systems biology of phenotypic robustness and plasticity. Integrative and Comparative Biology 57:171-184.

Ostrowski, E. A., C. Ofria, and R. E. Lenski. 2015. Genetically integrated traits and rugged adaptive landscapes in digital organisms. BMC Evolutionary Biology 15:83.

Paudel, B. B., and V. Quaranta. 2019. Metabolic plasticity meets gene regulation. Proceedings of the National Academy of Sciences of the USA 116:3370-3372.

Payen, V. L., P. E. Porporato, B. Baselet, and P. Sonveaux. 2016. Metabolic changes associated with tumor metastasis, part 1: Tumor $\mathrm{pH}$, glycolysis and the pentose phosphate pathway. Cellular and Molecular Life Sciences 73:1333-1348.

Pelosi, L., L. Kühn, D. Guetta, J. Garin, J. Geiselmann, R. E. Lenski, and D. Schneider. 2006. Parallel changes in global protein profiles during long-term experimental evolution in Escherichia coli. Genetics 173:1851-1869.

Philippe, N., E. Crozat, R. E. Lenski, and D. Schneider. 2007. Evolution of global regulatory networks during a long-term experiment with Escherichia coli. BioEssays 29:846-860.

Plucain, J., T. Hindré, M. Le Gac, O. Tenaillon, S. Cruveiller, C. Médigue, N. Leiby, et al. 2014. Epistasis and allele specificity in the emergence of a stable polymorphism in Escherichia coli. Science 343:1366-1369.

Quandt, E. M., J. Gollihar, Z. D. Blount, A. D. Ellington, G. Georgiou, and J. E. Barrick. 2015. Fine-tuning citrate synthase flux potentiates and refines metabolic innovation in the Lenski evolution experiment. eLife 4:e09696.

Rodríguez, J. A., U. M. Marigorta, D. A. Hughes, N. Spataro, E. Bosch, and A. Navarro. 2017. Antagonistic pleiotropy and mutation accumulation influence human senescence and disease. Nature Ecology and Evolution 1:1-5.

Rozen, D. E., D. Schneider, and R. E. Lenski. 2005. Long-term experimental evolution in Escherichia coli. XIII. Phylogenetic history of a balanced polymorphism. Journal of Molecular Evolution 61:171-180. 
Ruiz, J. A., R. O. Fernández, P. I. Nikel, B. S. Méndez, and M. J. Pettinari. 2006. Dye (Arc) mutants: Insights into an unexplained phenotype and its suppression by the synthesis of poly (3-hydroxybutyrate) in Escherichia coli recombinants. FEMS Microbiology Letters 258:55-60.

Salmon, K. A., S. Hung, N. R. Steffen, R. Krupp, P. Baldi, G. W. Hatfield, and R. P. Gunsalus. 2005. Global gene expression profiling in Escherichia coli K12: effects of oxygen availability and ArcA. Journal of Biological Chemistry 280:15084-15096.

Saxer, G., M. D. Krepps, E. D. Merkley, C. Ansong, B. L. Deatherage Kaiser, M. T. Valovska, N. Ristic, et al. 2014. Mutations in global regulators lead to metabolic selection during adaptation to complex environments. PLoS Genetics 10:e1004872.

Setlow, P. 2006. Spores of Bacillus subtilis: Their resistance to and killing by radiation, heat and chemicals. Journal of Applied Microbiology 101:514-525.

Shabalina, S. A., L. Y. Yampolsky, and A. S. Kondrashov. 1997. Rapid decline of fitness in panmictic populations of Drosophila melanogaster maintained under relaxed natural selection. Proceedings of the National Academy of Sciences of the USA 94:13034-13039.

Shannon, P., A. Markiel, O. Ozier, N. S. Baliga, J. T. Wang, D. Ramage, N. Amin, et al. 2003. Cytoscape: A software environment for integrated models of biomolecular interaction networks. Genome Research 13:2498-2504.

Shewaramani, S., T. J. Finn, S. C. Leahy, R. Kassen, P. B. Rainey, and C. D. Moon. 2017. anaerobically grown Escherichia coli has an enhanced mutation rate and distinct mutational spectra. PLoS Genetics 13:e1006570.

Siegal, M. L., and J. Leu. 2014. On the nature and evolutionary impact of phenotypic robustness mechanisms. Annual Review of Ecology, Evolution, and Systematics 45:495-517.

Sniegowski, P. D., P. J. Gerrish, and R. E. Lenski. 1997. Evolution of high mutation rates in experimental populations of E. coli. Nature 387:703-705.

Sokal, R. R., Rohlf, F. J. 1995. Biometry: The principles and practice of statistics in biological research, 3rd Edition. W. H. Freeman, New York.

Soo, R. M., J. Hemp, D. H. Parks, W. W. Fischer, and P. Hugenholtz. 2017. On the origins of oxygenic photosynthesis and aerobic respiration in Cyanobacteria. Science 355:1436-1440.

Stirling, F., L. Bitzan, S. O’Keefe, E. Redfield, J. W. K. Oliver, J. Way, and P. A. Silver. 2017. Rational design of evolutionarily stable microbial kill switches. Molecular Cell 68:686-697.

Swain, A., and W. F. Fagan. 2019. A mathematical model of the Warburg Effect: Effects of cell size, shape and substrate availability on growth and metabolism in bacteria. Mathematical Biosciences and Engineering 16:168-186.

Szklarczyk, D., J. H. Morris, H. Cook, M. Kuhn, S. Wyder, M. Simonovic, A. Santos, et al. 2017. The STRING database in 2017: Quality-controlled protein-protein association networks, made broadly accessible. Nucleic Acids Research 45:D362-D368.

Tenaillon, O., J. E. Barrick, N. Ribeck, D. E. Deatherage, J. L. Blanchard, A. Dasgupta, G. C. Wu, et al. 2016. Tempo and mode of genome evolution in a 50,000-generation experiment. Nature 536:165-170.

Travisano, M., and R. E. Lenski. 1996. Long-term experimental evolution in Escherichia coli. IV. 
Targets of selection and the specificity of adaptation. Genetics 143:15-26.

Unden, G., and J. Bongaerts. 1997. Alternative respiratory pathways of Escherichia coli: energetics and transcriptional regulation in response to electron acceptors. Biochimica et Biophysica Acta 1320:217-234.

Unden, G., S. Becker, J. Bongaerts, J. Schirawski, and S. Six. 1994. Oxygen regulated gene expression in facultatively anaerobic bacteria. Antonie van Leeuwenhoek 66:3-22.

Van den Bergh, B., T. Swings, M. Fauvart, and J. Michiels. 2018. Experimental design, population dynamics, and diversity in microbial experimental evolution. Microbiology and Molecular Biology Reviews 82:e00008-18.

Vasi, F., M. Travisano, and R. E. Lenski. 1994. Long-term experimental evolution in Escherichia coli. II. Changes in life-history traits during adaptation to a seasonal environment. American Naturalist 144:432-456.

Wagner, G. P., and J. Mezey. 2000. Modeling the evolution of genetic architecture: A continuum of alleles model with pairwise AxA epistasis. Journal of Theoretical Biology 203:163-175.

Warburg, O. 1956. On the origin of cancer cells. Science 123:309-314.

Wielgoss, S., J. E. Barrick, O. Tenaillon, M. J. Wiser, W. J. Dittmar, S. Cruveiller, B. ChaneWoon-Ming, et al. 2013. Mutation rate dynamics in a bacterial population reflect tension between adaptation and genetic load. Proceedings of the National Academy of Sciences of the USA 110:222-227.

Williams, G. C. 1957. Pleiotropy, natural selection, and the evolution of senescence. Evolution $11: 398-411$.

Wiser, M. J., N. Ribeck, and R. E. Lenski. 2013. Long-term dynamics of adaptation in asexual populations. Science 342:1364-1367.

Woods, R., D. Schneider, C. L. Winkworth, M. A. Riley, and R. E. Lenski. 2006. Tests of parallel molecular evolution in a long-term experiment with Escherichia coli. Proceedings of the National Academy of Sciences of the USA 103:9107-9112. 
877 Table 1: ANOVAs of relative fitness for clones sampled from the LTEE populations at three 878 timepoints and measured in either the oxic or anoxic environment

\begin{tabular}{|c|c|c|c|c|c|c|c|}
\hline \multirow[b]{2}{*}{ Generation } & \multirow[b]{2}{*}{ Source } & \multicolumn{3}{|c|}{ Oxic } & \multicolumn{3}{|c|}{ Anoxic } \\
\hline & & df & $F$ & $p$ & df & $F$ & $p$ \\
\hline \multirow{2}{*}{2,000} & Lineage & 10 & 1.0441 & 0.4246 & 10 & 3.6004 & 0.0016 \\
\hline & Error & 43 & & & 42 & & \\
\hline \multirow{2}{*}{10,000} & Lineage & 10 & 1.1091 & 0.3770 & 7 & 0.9220 & 0.5225 \\
\hline & Error & 44 & & & 31 & & \\
\hline \multirow{2}{*}{50,000} & Lineage & 8 & 3.9393 & 0.0022 & 5 & 2.7139 & 0.0194 \\
\hline & Error & 34 & & & 24 & & \\
\hline
\end{tabular}

879 Note: Eleven lineages were examined at 2,000 and 10,000 generations, but only 9 lineages at

88050,000 generations. The fitness assays were replicated 5-fold for each clone, but technical errors 881 resulted in a few missing values. 


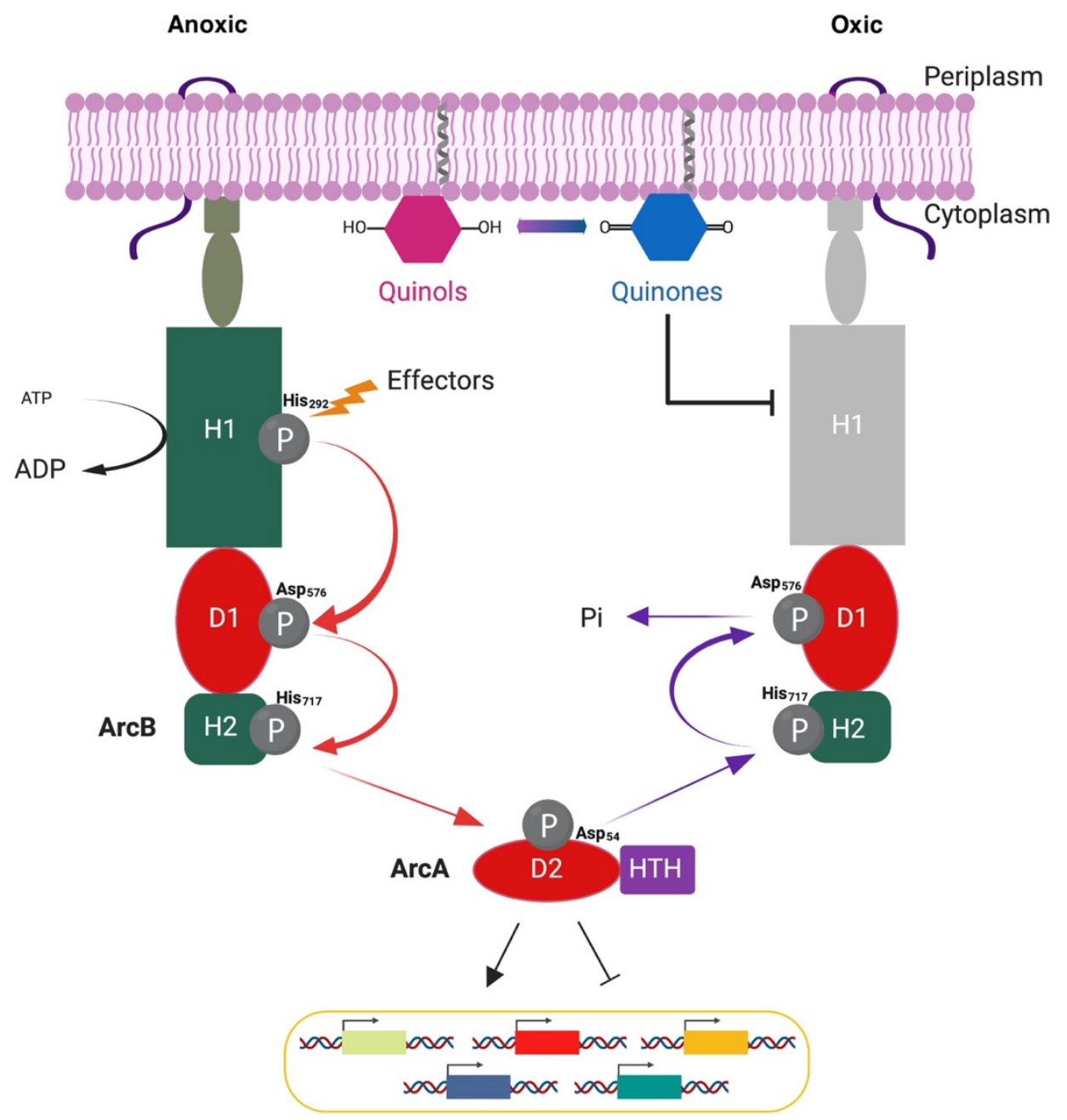

Arc Modulon

885 Figure 1: Schematic representation of ArcAB-dependent gene regulation. In an anoxic environment, the transmembrane sensor kinase ArcB undergoes autophosphorylation. This reaction is enhanced by fermentation metabolites such as $\mathrm{D}$-lactate, pyruvate and acetate that act as effectors. Three conserved residues (His 292, Asp $_{576}$, His717) in ArcB sequentially transfer the

889 phosphoryl group onto ArcA, the response regulator, at a conserved Asp 54 residue. Phosphorylated 890 ArcA (ArcA-P), in turn, represses the transcription of many operons involved in respiratory 891 metabolism, while activating those encoding proteins involved in fermentative metabolism. In an 892 oxic environment, $\mathrm{ArcB}$ autophosphorylation ceases and $\mathrm{ArcA}$ is dephosphorylated by reversing 893 the phosphorelay, leading to the release of inorganic phosphate into the cytoplasm. Figure adapted 894 from Kwon et al. (2003). 


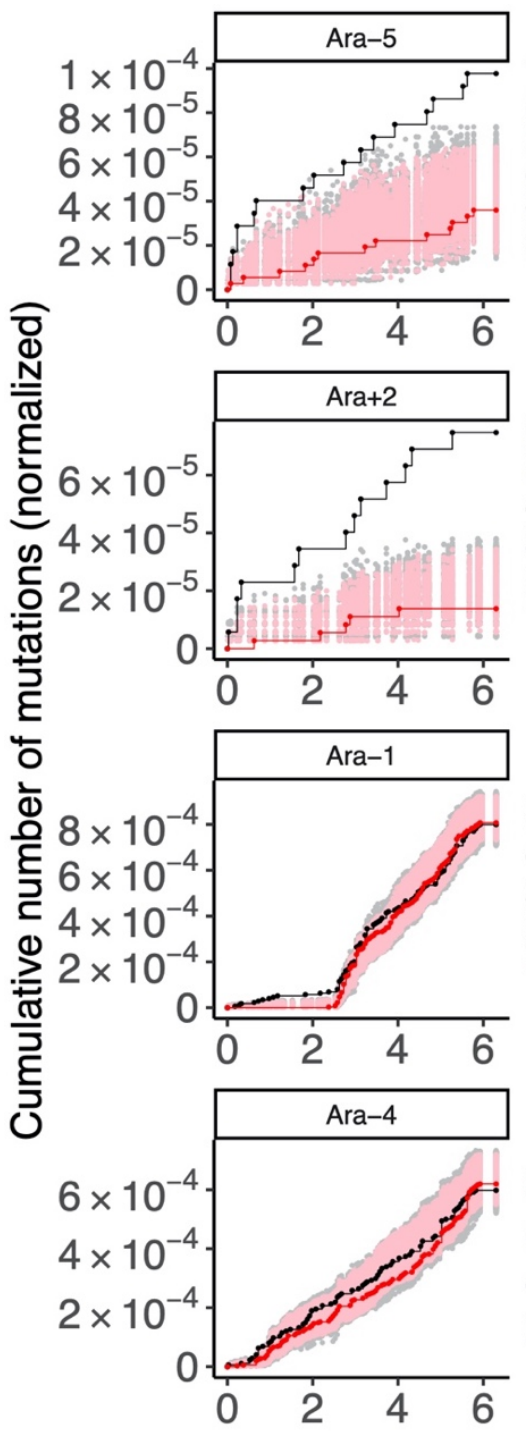

895

896

897

Figure 2: Cumulative numbers of mutations in aerobic- and anaerobic-specific genes in the LTEE whole-population samples. Each panel shows the number of nonsynonymous mutations in aerobic(black) and anaerobic-specific (red) genes in the indicated population through 60,000 generations.
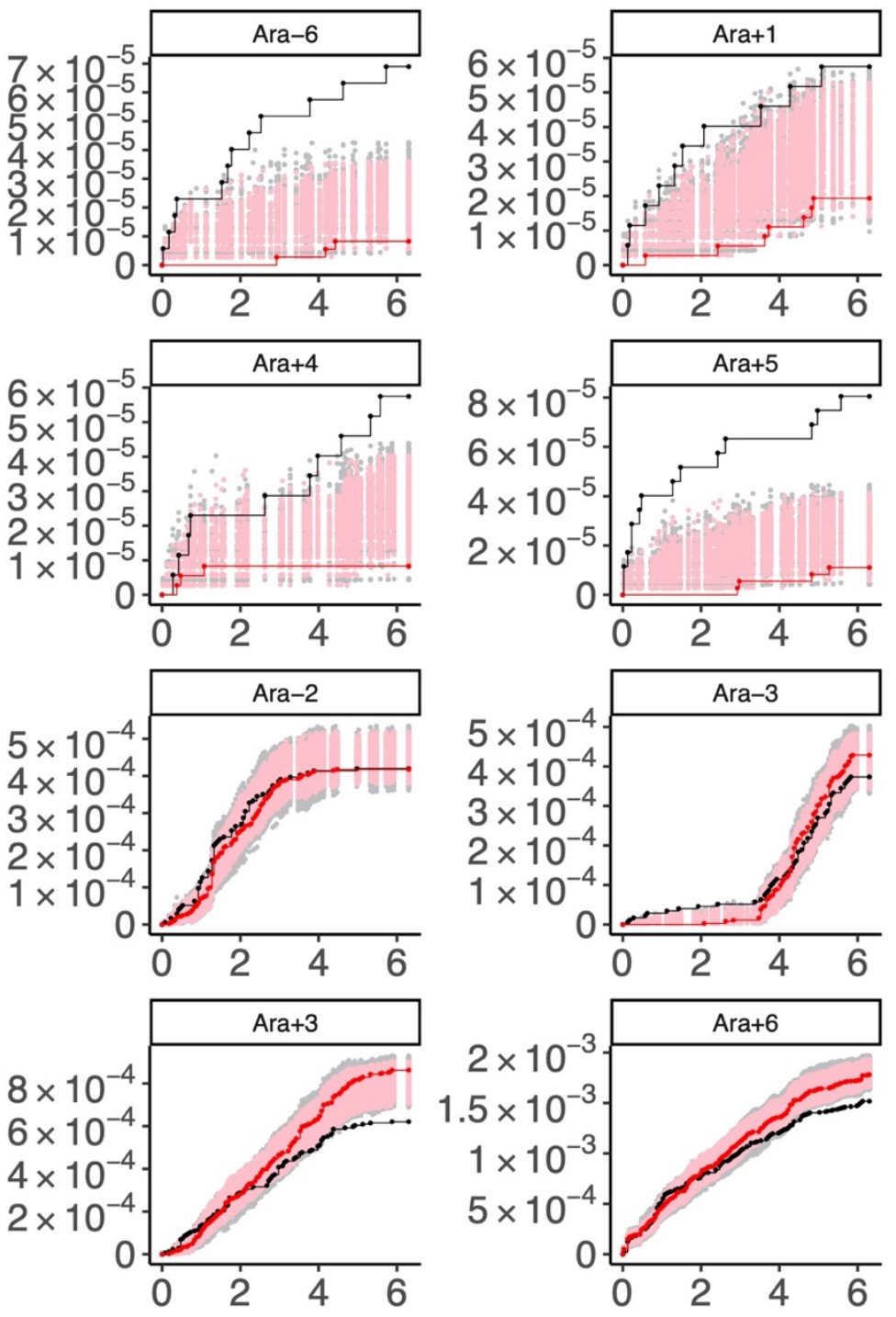

Generations (x 10,000)
For comparison, random sets of genes of equal cardinality to the aerobic- (227) or anaerobicspecific (345) gene sets were sampled 1,000 times, and the cumulative number of nonsynonymous mutations was calculated to generate a null distribution of the expected number of mutations for each population. The gray and pink points show $95 \%$ of these null distributions (excluding $2.5 \%$ in each tail) for aerobic and anaerobic comparisons, respectively. 


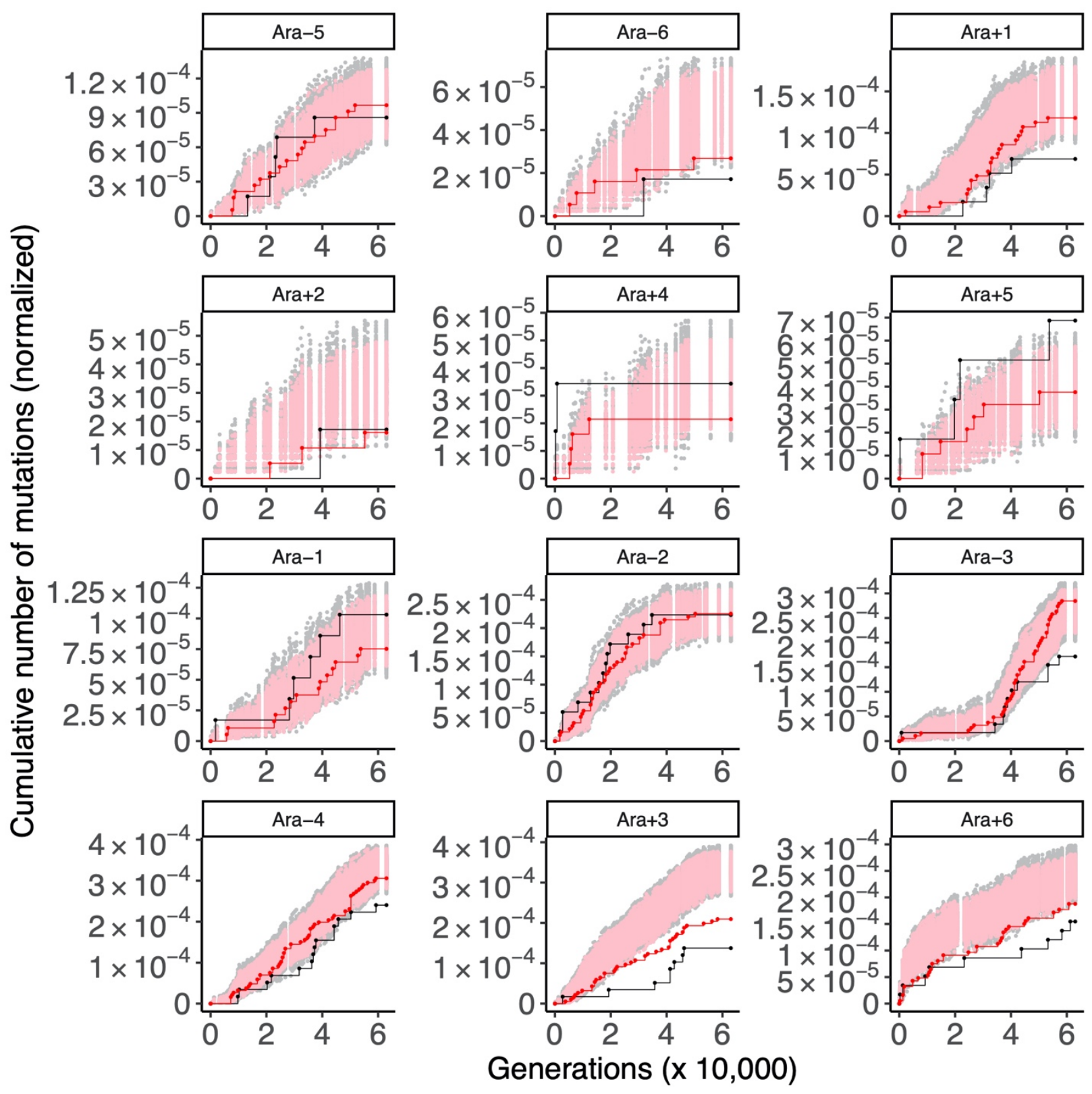

908 Figure 3: Cumulative numbers of indel, nonsense, and structural mutations in protein-coding

909 genes in the LTEE whole-population samples. The black and red points are mutations in aerobic-

910 and anaerobic-specific genes, respectively. The gray and pink points show the corresponding null

911 distributions based on randomized gene sets. See Figure 2 for additional details.

912 
913

A
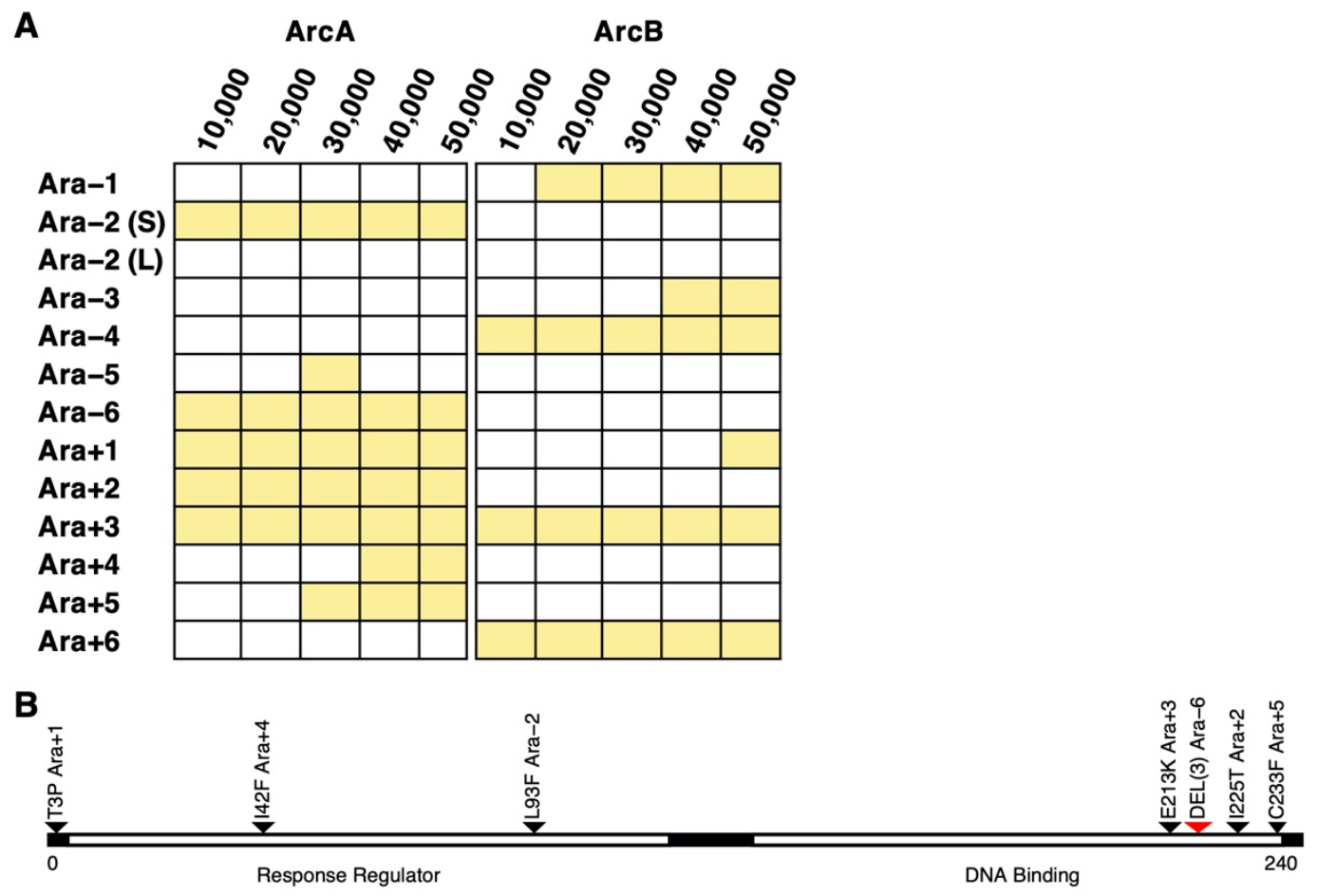

C

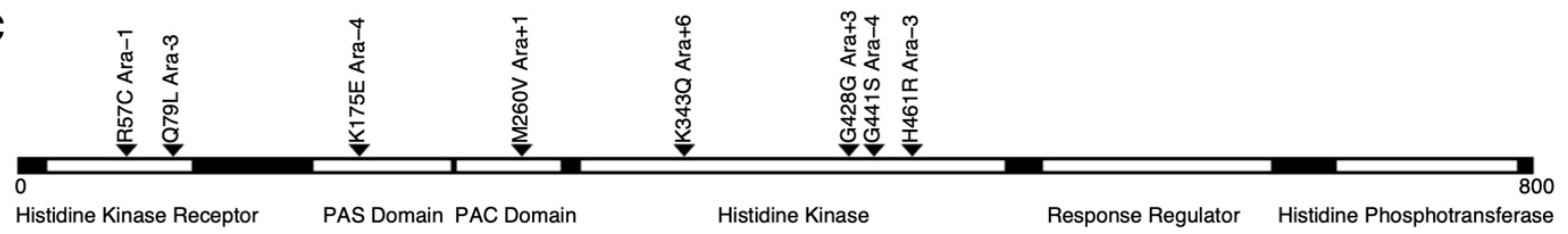

916

917 Figure 4: Mutations in $\operatorname{arc} A$ and $\operatorname{arcB}$ in clones from the LTEE. (A) Shading indicates that the

918 clone has a mutation in $\operatorname{arc} A$ or $\operatorname{arcB}$, which encode proteins ArcA and ArcB, respectively. Clones

919 from two coexisting lineages, labeled S and L, are shown for population Ara-2. Sequence data are

920 from Tenaillon et al. (2016), with one exception: Plucain et al. (2014) show that the $\operatorname{arc} A$ mutation

921 had already fixed in the Ara-2 S lineage by 10,000 generations. Mutations present in the 50,000-

922 generation clones are mapped onto the functional domains of the (B) ArcA and (C) ArcB proteins.

923 The red arrow marks a deletion of 3 amino acids; all other mutations are point mutations resulting 924 in amino-acid substitutions. 


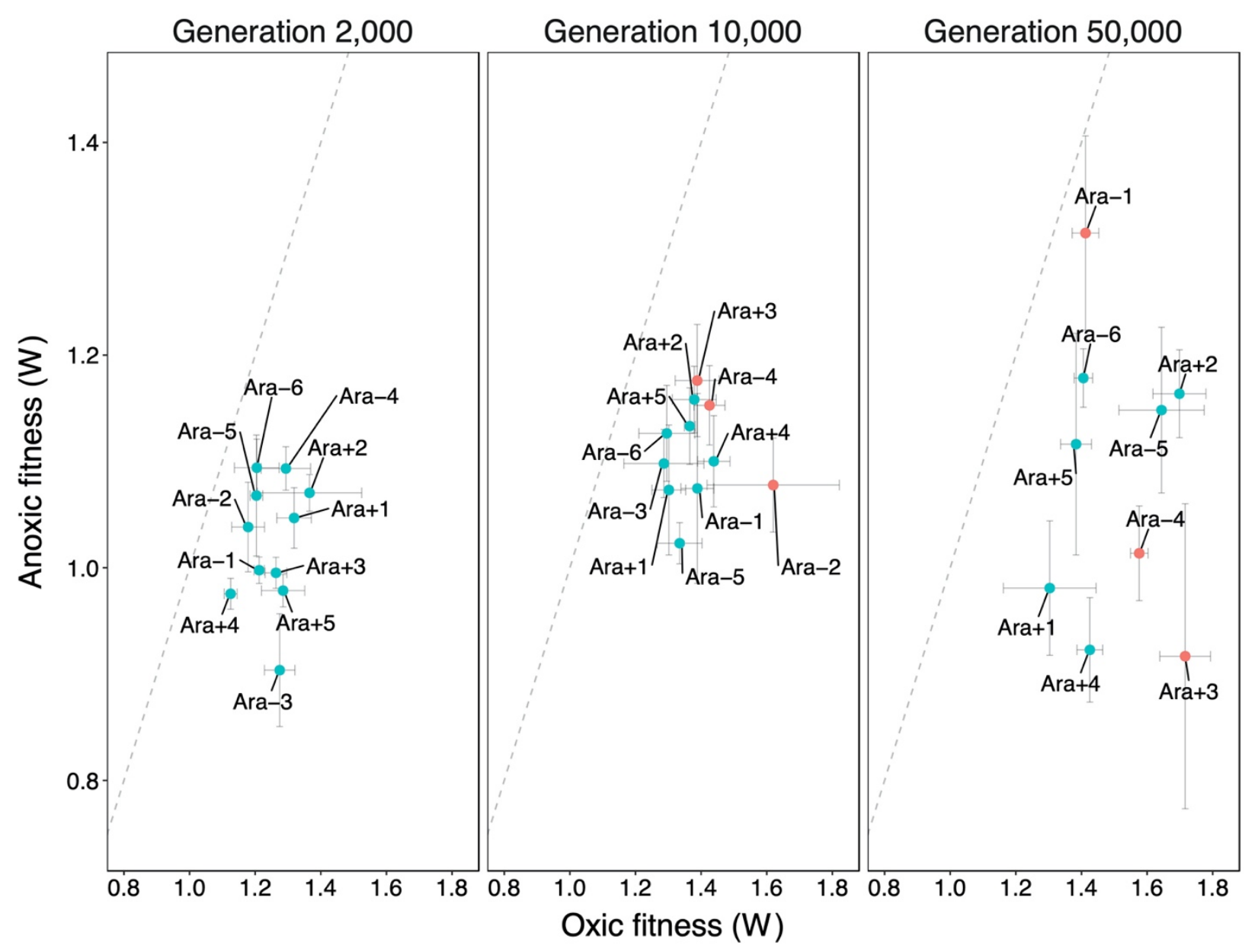

928 Figure 5: Evolved clones are better adapted to the aerobic environment in which they evolved

929 than to the novel, but otherwise identical, anoxic environment. Each point is the mean fitness for

930 an evolved clone sampled from the indicated population at three different generations, measured

931 relative to the LTEE ancestor with the opposite marker state. Orange and teal points indicate that

932 the corresponding population had or had not evolved hypermutability, respectively. Error bars

933 show the standard errors based on replicated fitness assays in each environment for each clone. 


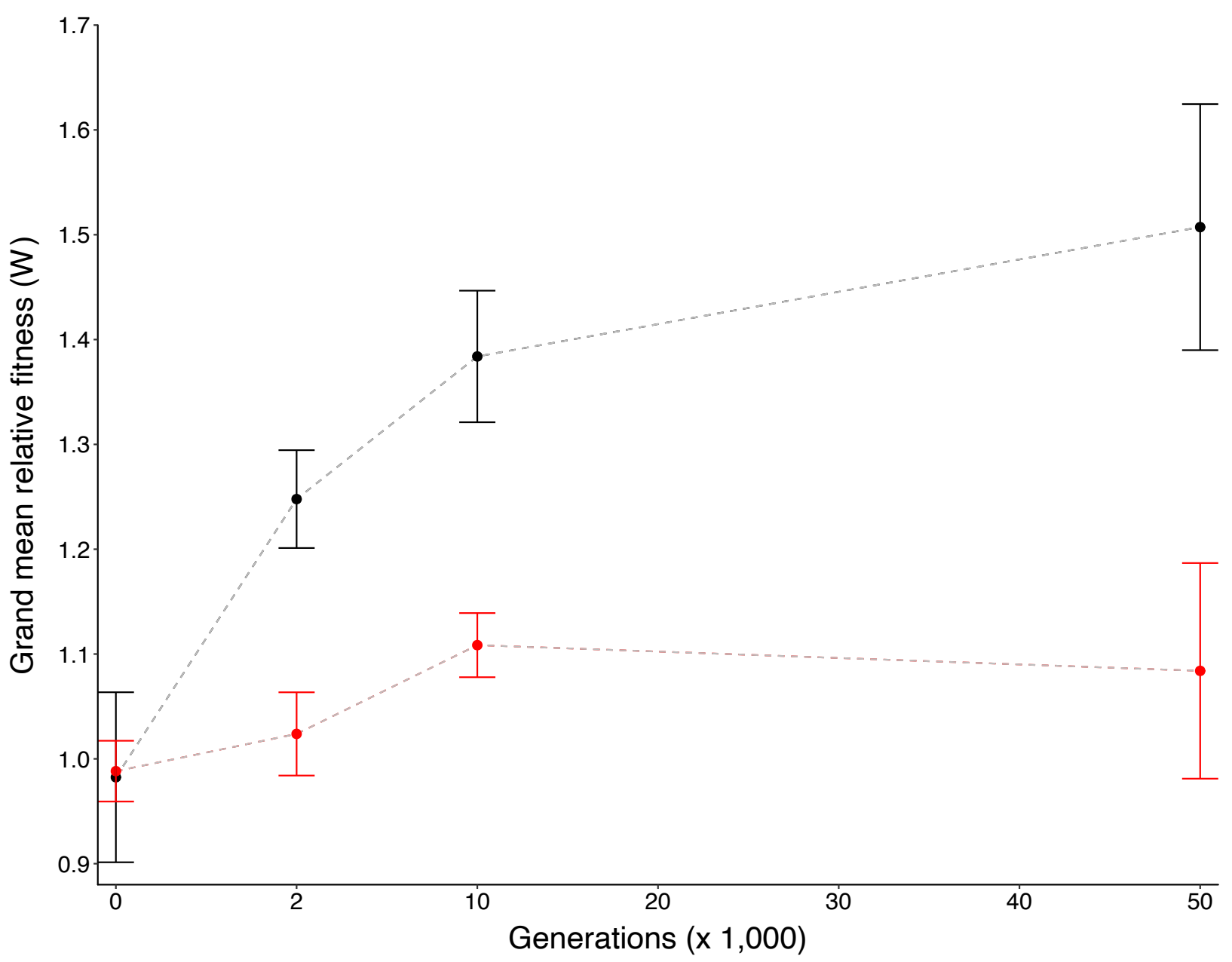

938 Figure 6: Relative fitness trajectories during 50,000 generations of evolution in an oxic 939 environment. Each point is the grand mean fitness of evolved clones sampled at 2,000, 10,000, 940 and 50,000 generations relative to the LTEE ancestors. Black and red symbols correspond to 941 fitnesses measured in the oxic and anoxic environments, respectively. Error bars show the $95 \%$ 942 confidence intervals based on 11, 11, and 9 assayed populations at 2,000, 10,000, and 50,000 943 generations, respectively. 
945

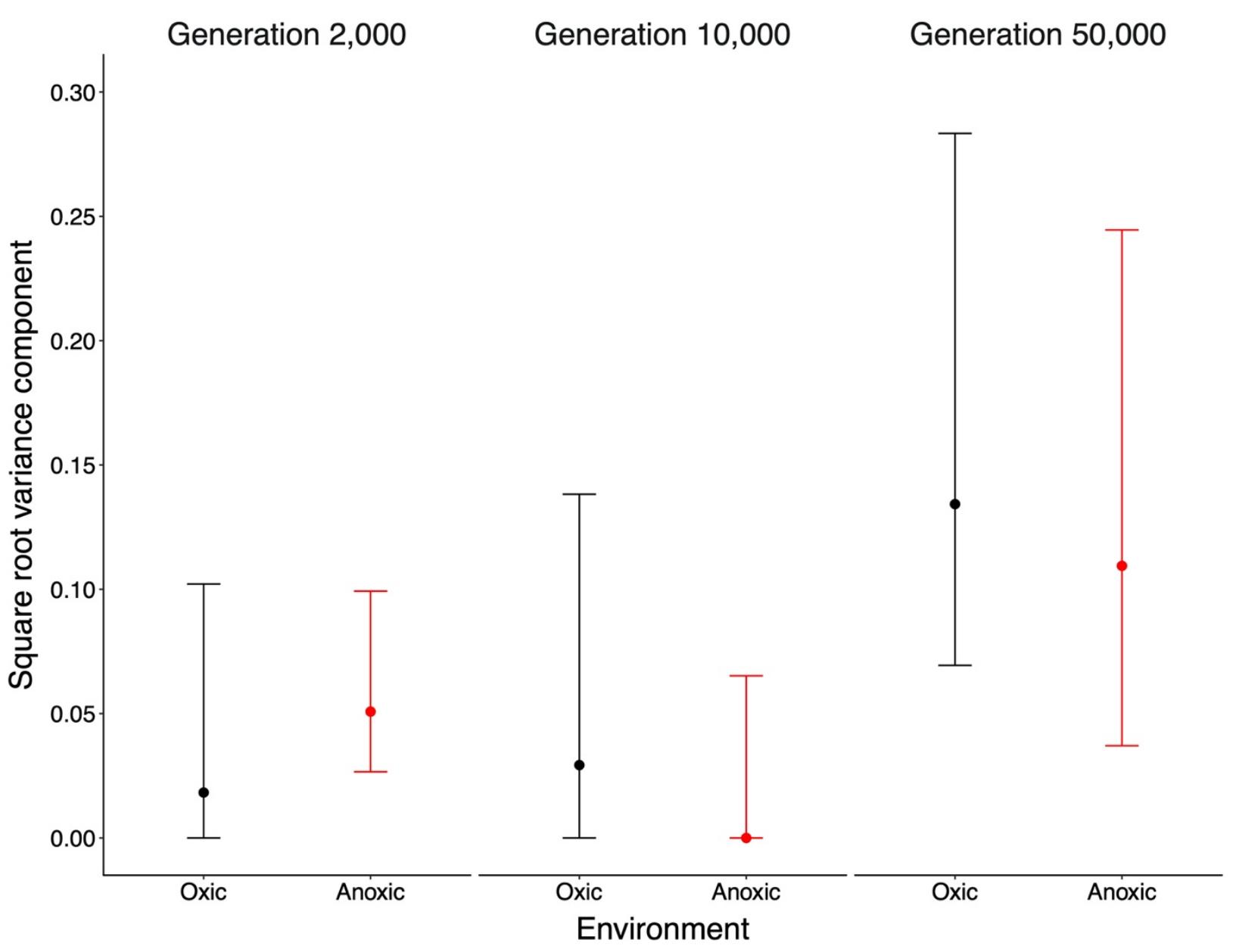

948 Figure 7: Among-population variance component for fitness in the oxic and anoxic environments.

949 Each point is the square root of the variance component estimated from a corresponding random-

950 effects ANOVA. Error bars are approximate 95\% confidence intervals obtained using the 951 Moriguti-Bulmer procedure (Sokal and Rohlf 1995). Negative values of estimates and confidence 952 limits are truncated at 0 , the lower theoretical bound. 


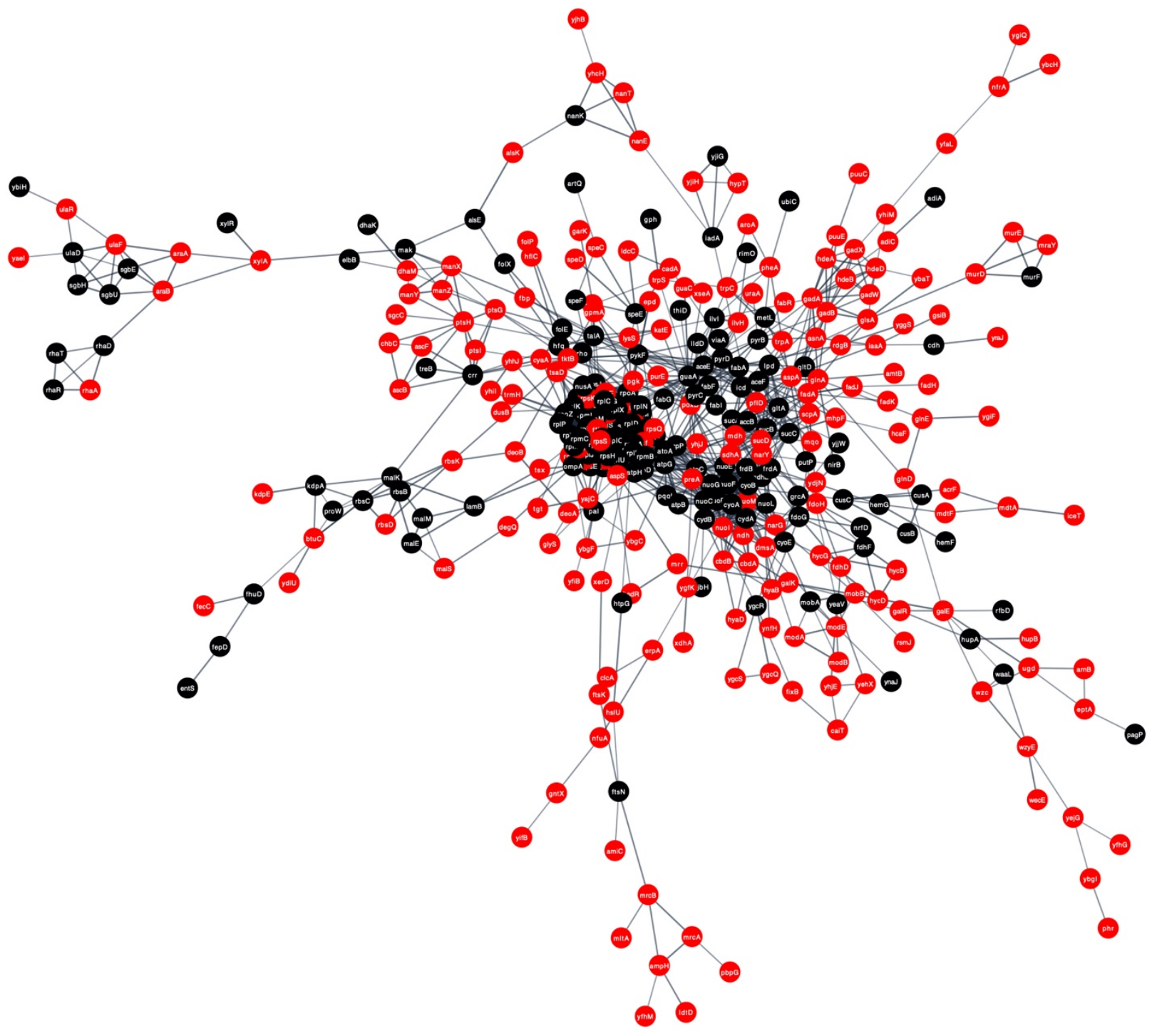

957 Figure 8: Network topology of aerobic- and anaerobic-specific genes. We used Cytoscape 958 (Shannon et al. 2003) to visualize protein-protein interactions between aerobic- (black) and 959 anerobic-specific (red) genes predicted by STRING (Szklarczyk et al. 2017). Each edge reflects a 960 confidence score of at least 0.7 (i.e., high) that an interaction exists, obtained using a maximum 961 likelihood approach and seven lines of evidence. In the supplemental PDF, Fig. S6 shows 962 additional genes that are not integrated within the main network shown here. 
Supplemental information for the paper:

\title{
Maintenance of Metabolic Plasticity Despite Relaxed Selection in a Long-Term Evolution Experiment with Escherichia coli
}

\author{
Nkrumah A. Grant, ${ }^{1,2,3 *}$ Rohan Maddamsetti, ${ }^{4}$ and Richard E. Lenski ${ }^{1,2,3}$
}

1. BEACON Center for the Study of Evolution in Action, Michigan State University, East Lansing, Michigan 48824; 2. Department of Microbiology and Molecular Genetics, Michigan State University, East Lansing, Michigan 48824; 3. Program in Ecology, Evolutionary Biology and Behavior, Michigan State University, East Lansing 48824; 4. Department of Biological Sciences, Old Dominion University, Norfolk, Virginia 23508.

* Corresponding author; e-mail: grantnkr@msu.edu 
bioRxiv preprint doi: https://doi.org/10.1101/2020.06.02.130138; this version posted June 3, 2020. The copyright holder for this preprint (which was not certified by peer review) is the author/funder, who has granted bioRxiv a license to display the preprint in perpetuity. It is made available under aCC-BY-NC-ND 4.0 International license.

Figure S1 (panels A-C)
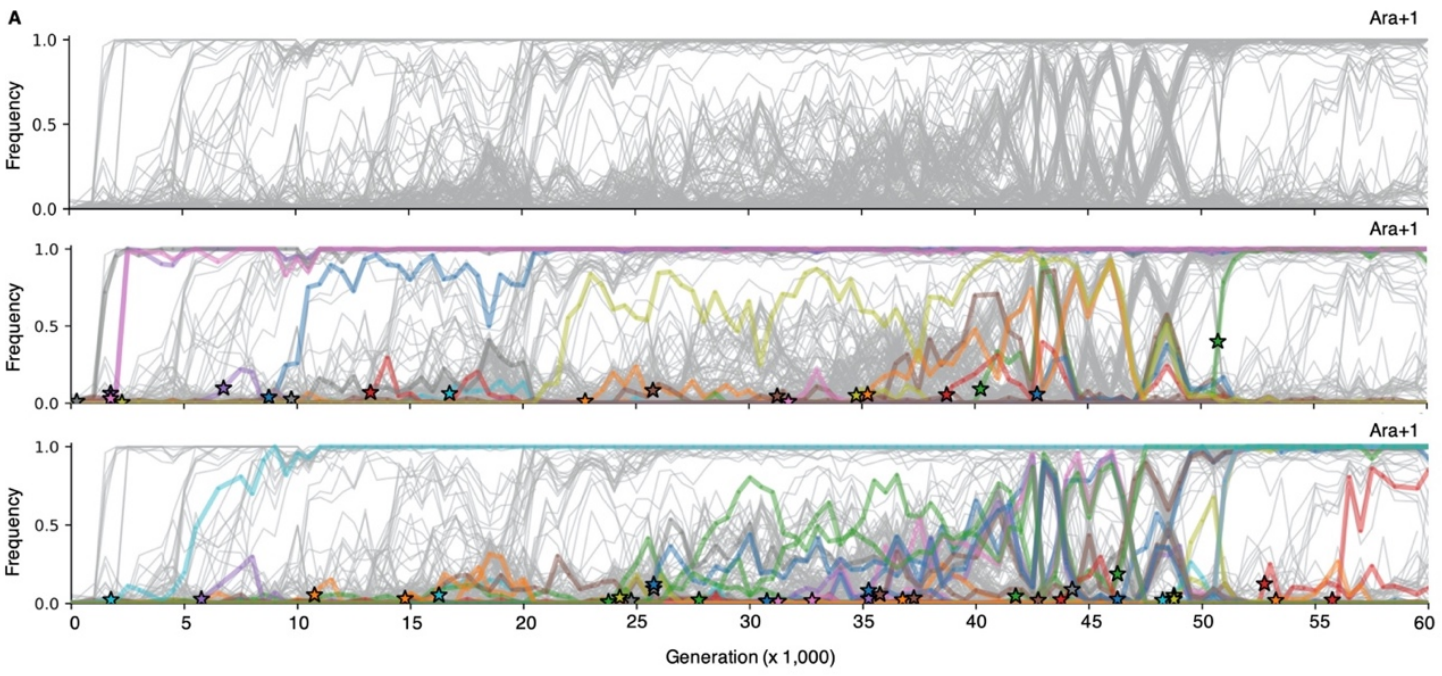

B
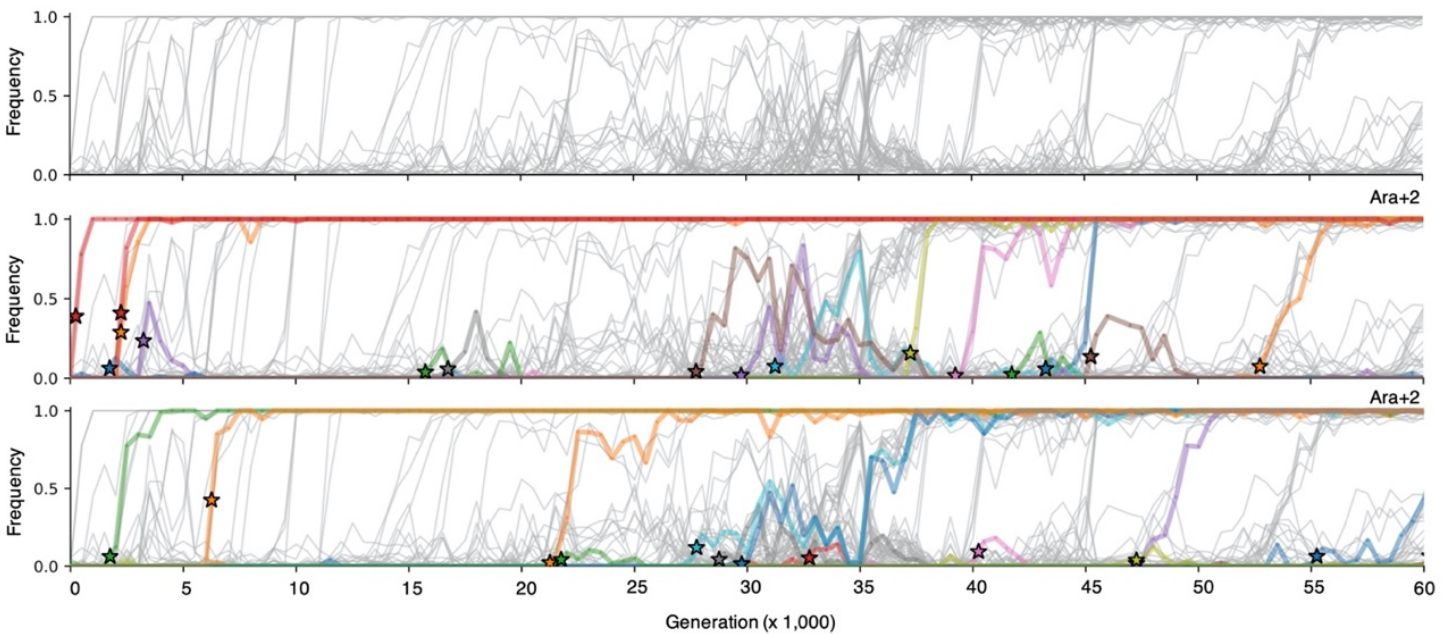

c
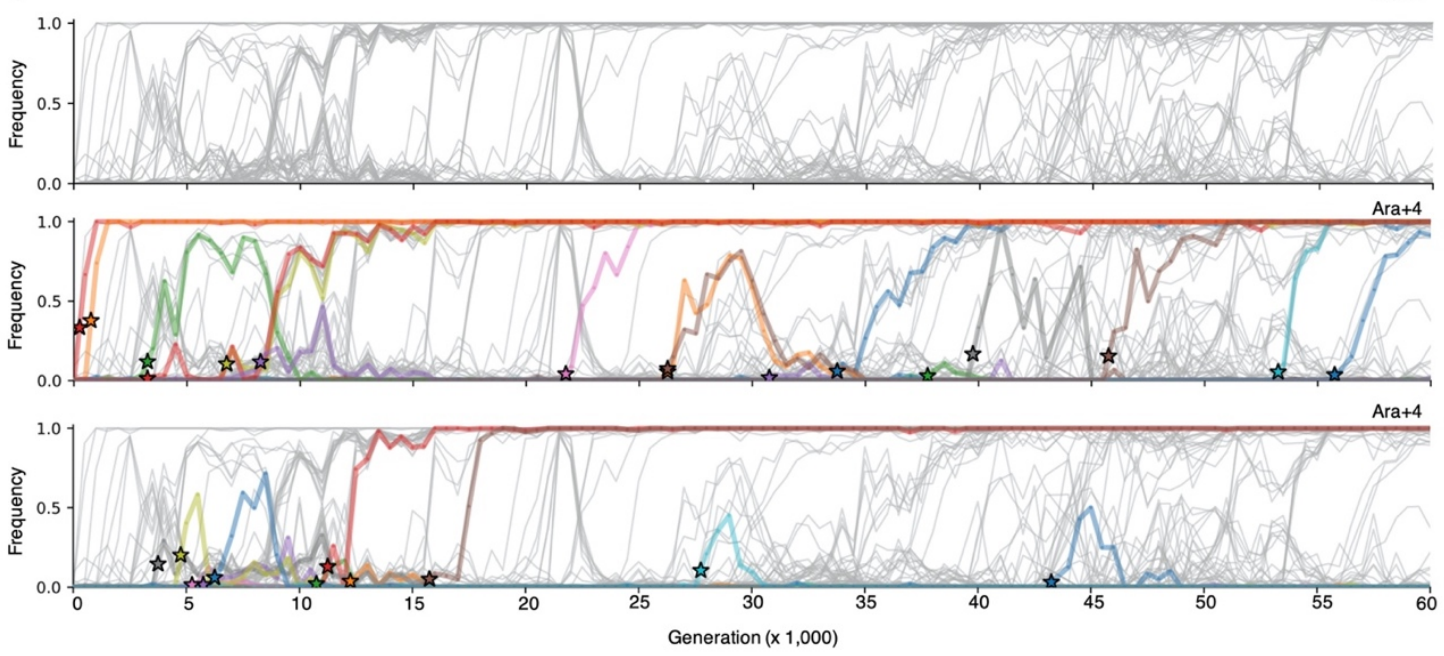
bioRxiv preprint doi: https://doi.org/10.1101/2020.06.02.130138; this version posted June 3, 2020. The copyright holder for this preprint (which was not certified by peer review) is the author/funder, who has granted bioRxiv a license to display the preprint in perpetuity. It is made available under aCC-BY-NC-ND 4.0 International license.

\section{Figure S1 (panels D-F)}

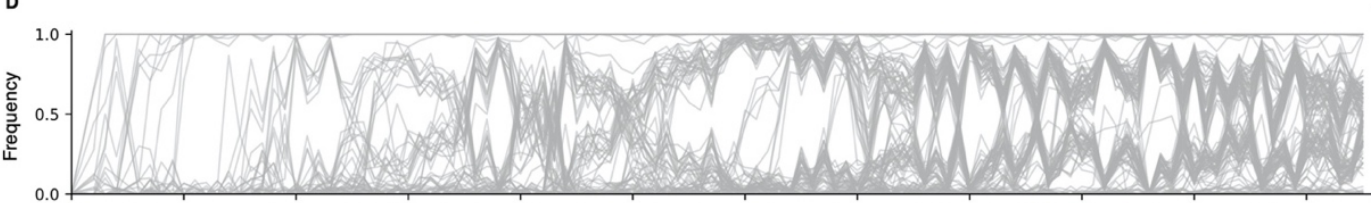

Ara +5
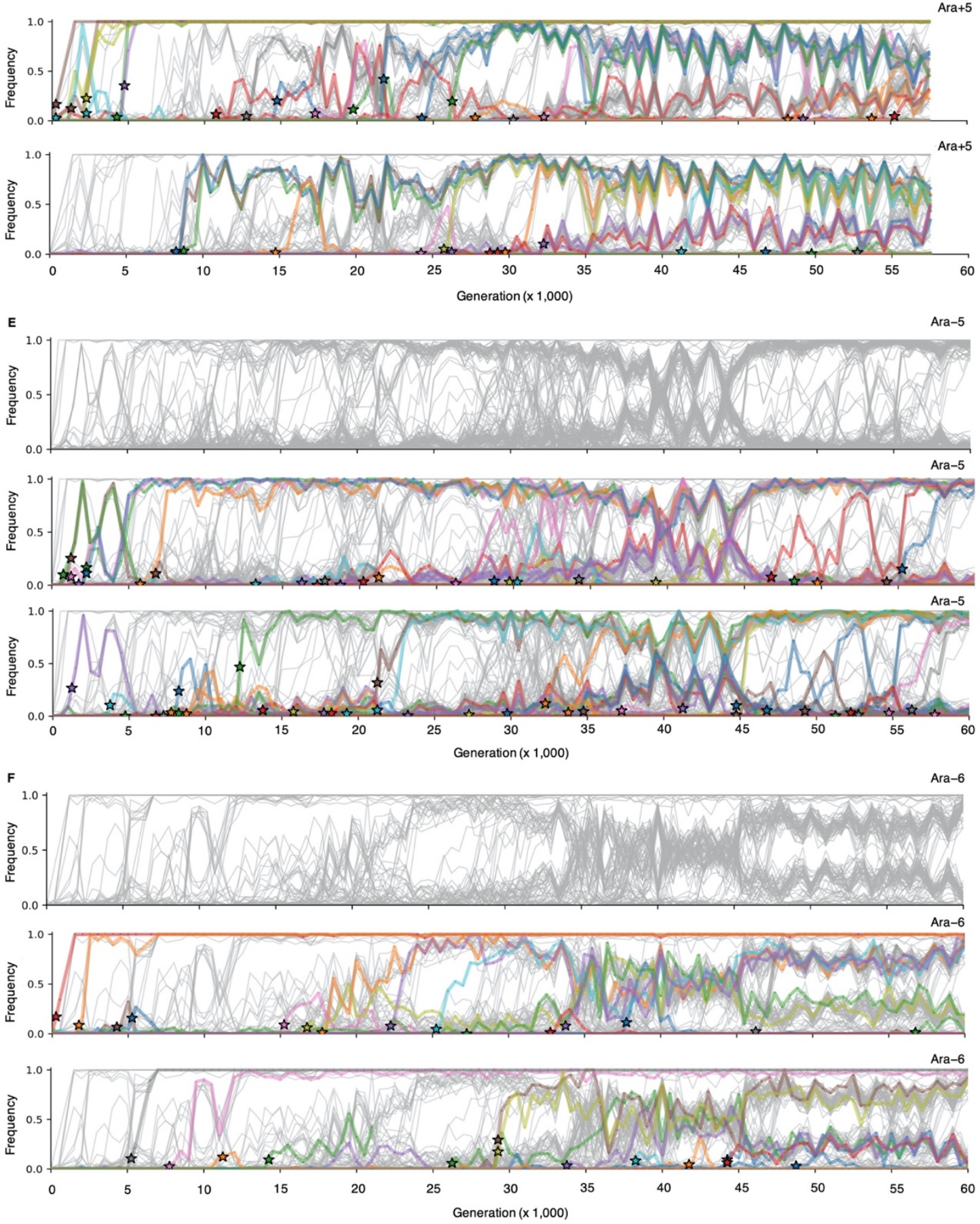
bioRxiv preprint doi: https://doi.org/10.1101/2020.06.02.130138; this version posted June 3, 2020. The copyright holder for this preprint (which was not certified by peer review) is the author/funder, who has granted bioRxiv a license to display the preprint in perpetuity. It is made available under aCC-BY-NC-ND 4.0 International license.

\section{Figure S1 (panels G-I)}

G
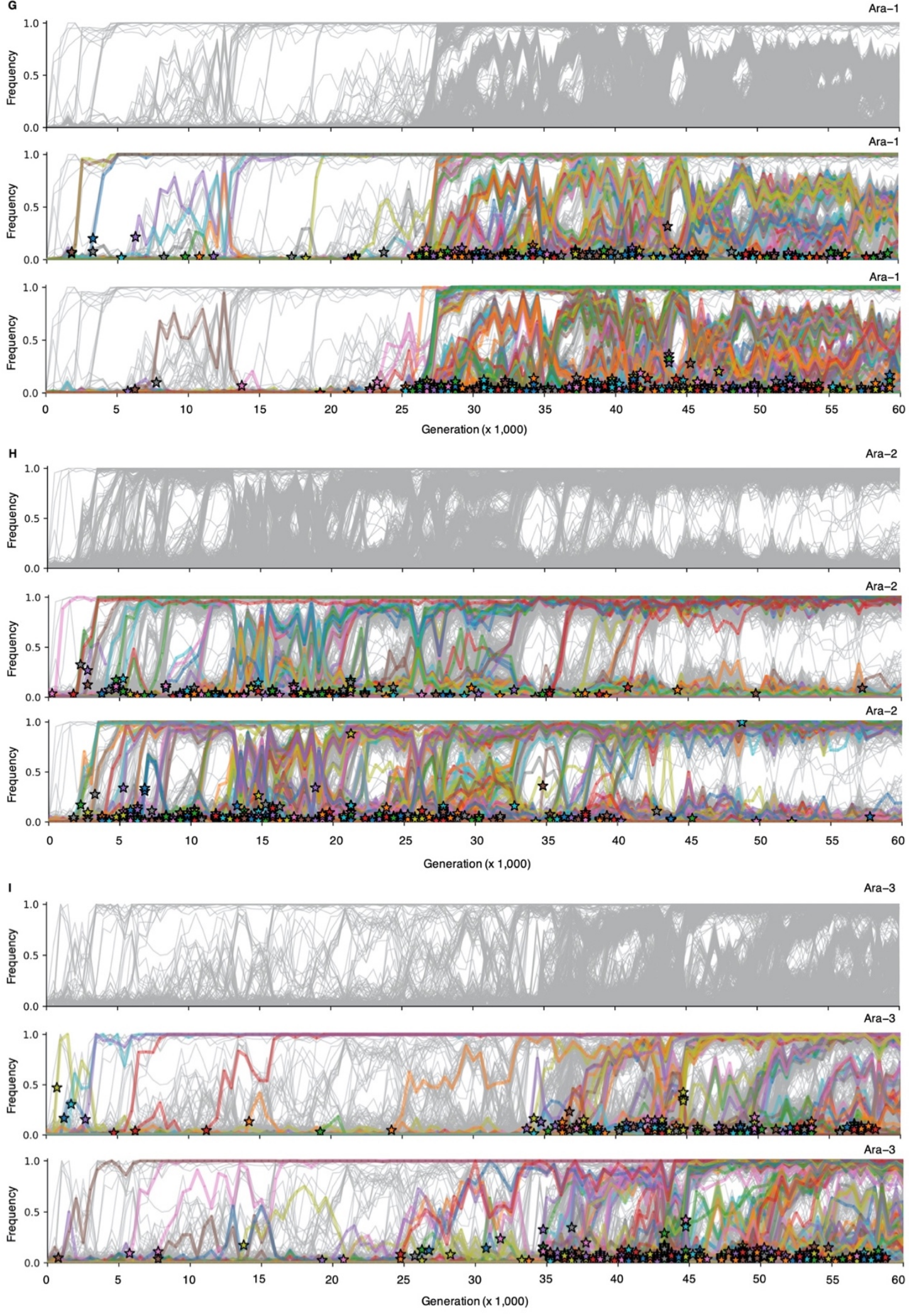
bioRxiv preprint doi: https://doi.org/10.1101/2020.06.02.130138; this version posted June 3, 2020. The copyright holder for this preprint (which was not certified by peer review) is the author/funder, who has granted bioRxiv a license to display the preprint in perpetuity. It is made available under aCC-BY-NC-ND 4.0 International license.

\section{Figure S1 (panels J-L)}
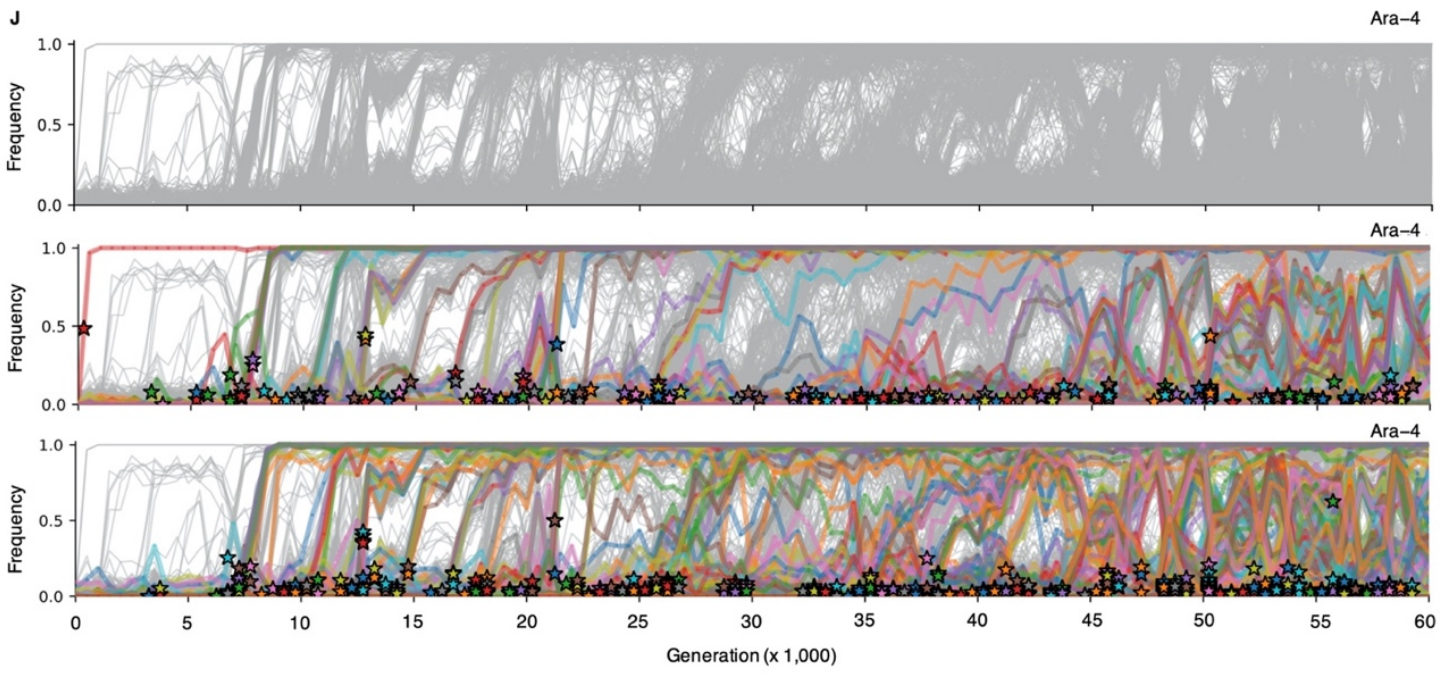

K
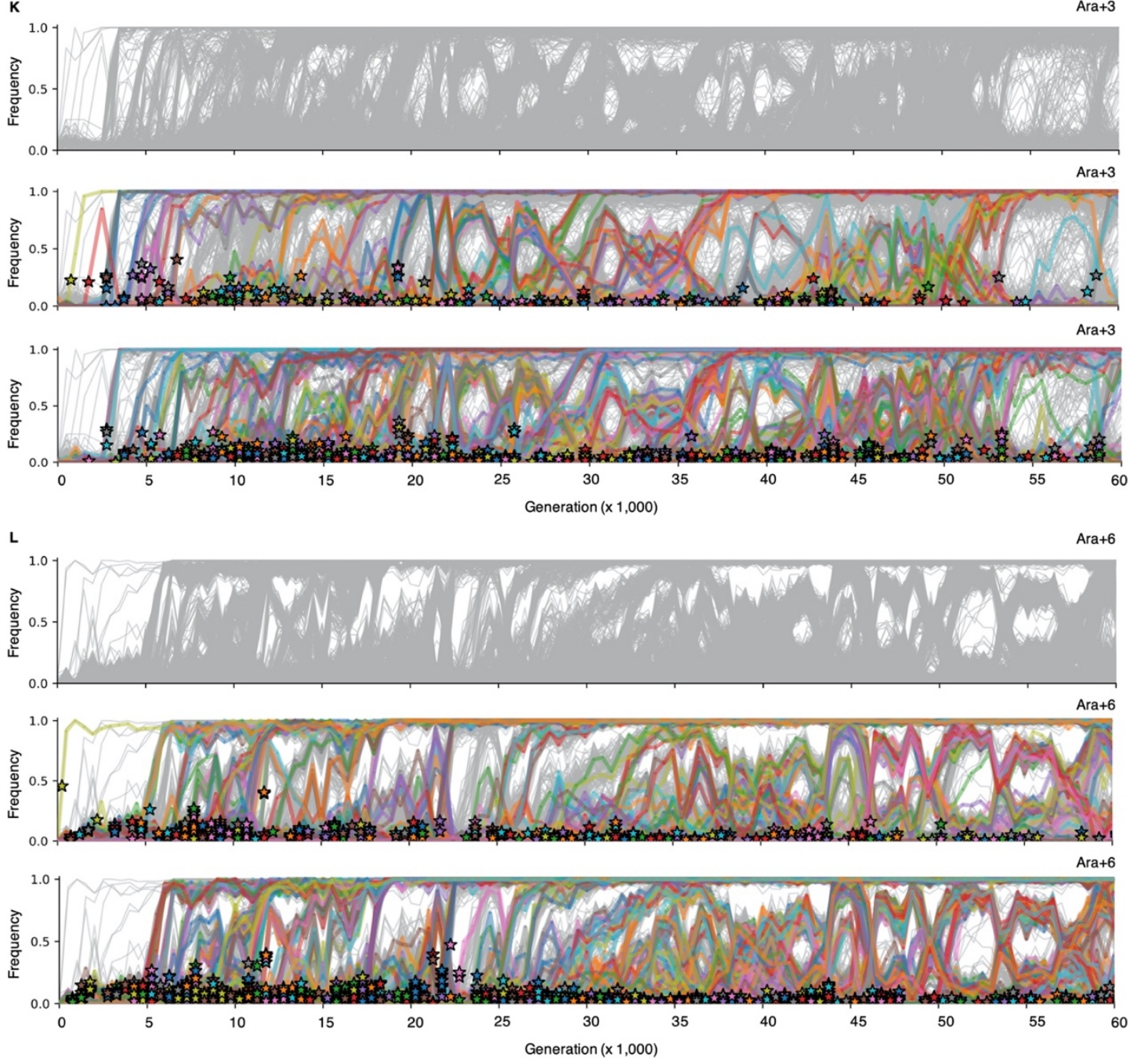
Figure S1: Evolutionary dynamics of mutations in aerobic-specific genes. Each panel (A-L) shows the allele-frequency trajectories for new mutations that arose in the indicated LTEE population and reached an observable frequency. Panels A-F show the six populations that never evolved hypermutability; panels G-L show the six populations that became mutators at various time points. In each panel, the top sub-panel shows in gray all observed mutations; the middle subpanel colors only those mutations in aerobic-specific genes; and the bottom sub-panel colors only those mutations in anaerobic-specific genes. The underlying metagenomic data and statistical criteria are from Good et al. (2017). The stars in the middle and bottom sub-panels mark the "appearance time" of mutations, which Good et al. (2017) defined as 250 generations before the first sample in which a given mutation reached observable frequency (i.e., the midpoint between that sample and the preceding sample, given 500 generations between successive samples). 


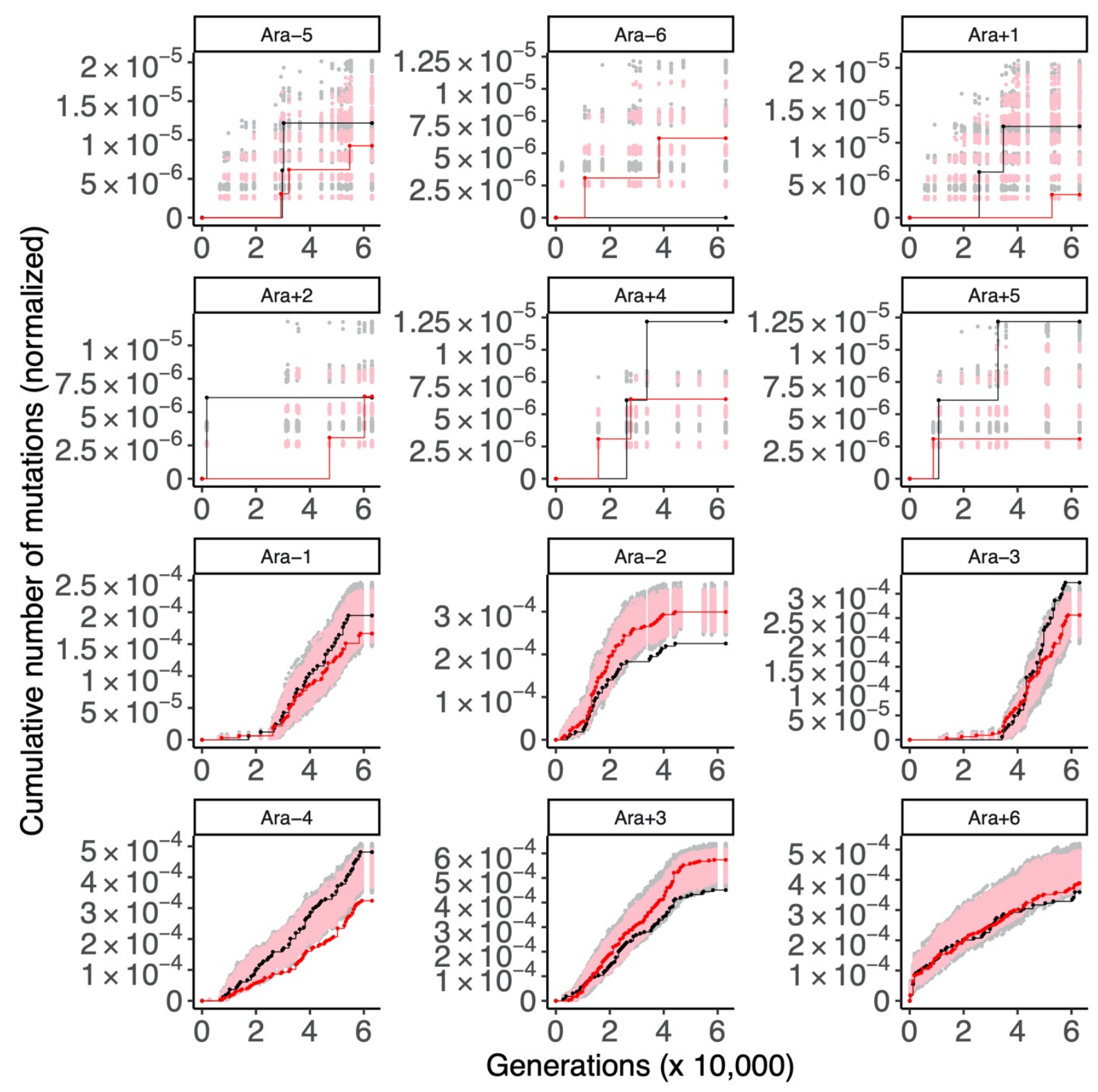

Figure S2: Cumulative number of mutations in aerobic- and anaerobic-specific genes in the LTEE whole-population samples. Each panel shows the number of synonymous mutations in aerobic(black) and anaerobic-specific (red) genes in the indicated population. For comparison, random sets of genes of equal cardinality to the aerobic- (227) or anaerobic-specific (345) gene sets were sampled 1,000 times, and the cumulative number of synonymous mutations was calculated to generate a null distribution of the expected number of mutations for each population. The gray and pink points show $95 \%$ of these null distributions (excluding $2.5 \%$ in each tail) for aerobic and anaerobic comparisons, respectively. 


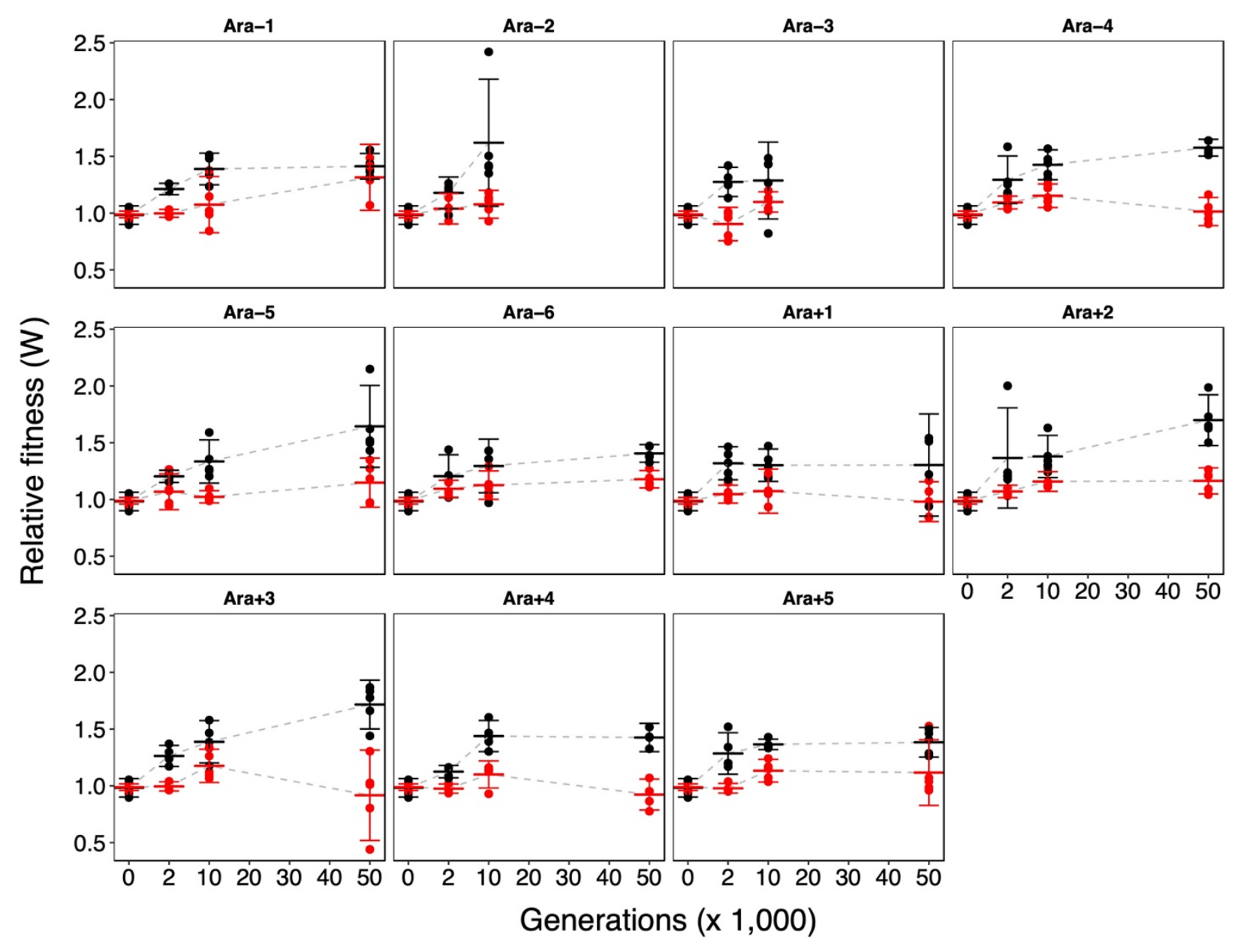

Figure S3: Relative fitness trajectories of individual LTEE populations in oxic and anoxic environments. Black and red points show competitions performed in oxic and anoxic environments, respectively. Each point is a replicate competition assay in which a clone from the indicated population was competed against the reciprocally marked ancestral strain. Two trajectories are truncated owing to technical difficulties (see Materials and Methods). Wide hash marks are means; error bars are $95 \%$ confidence intervals. 


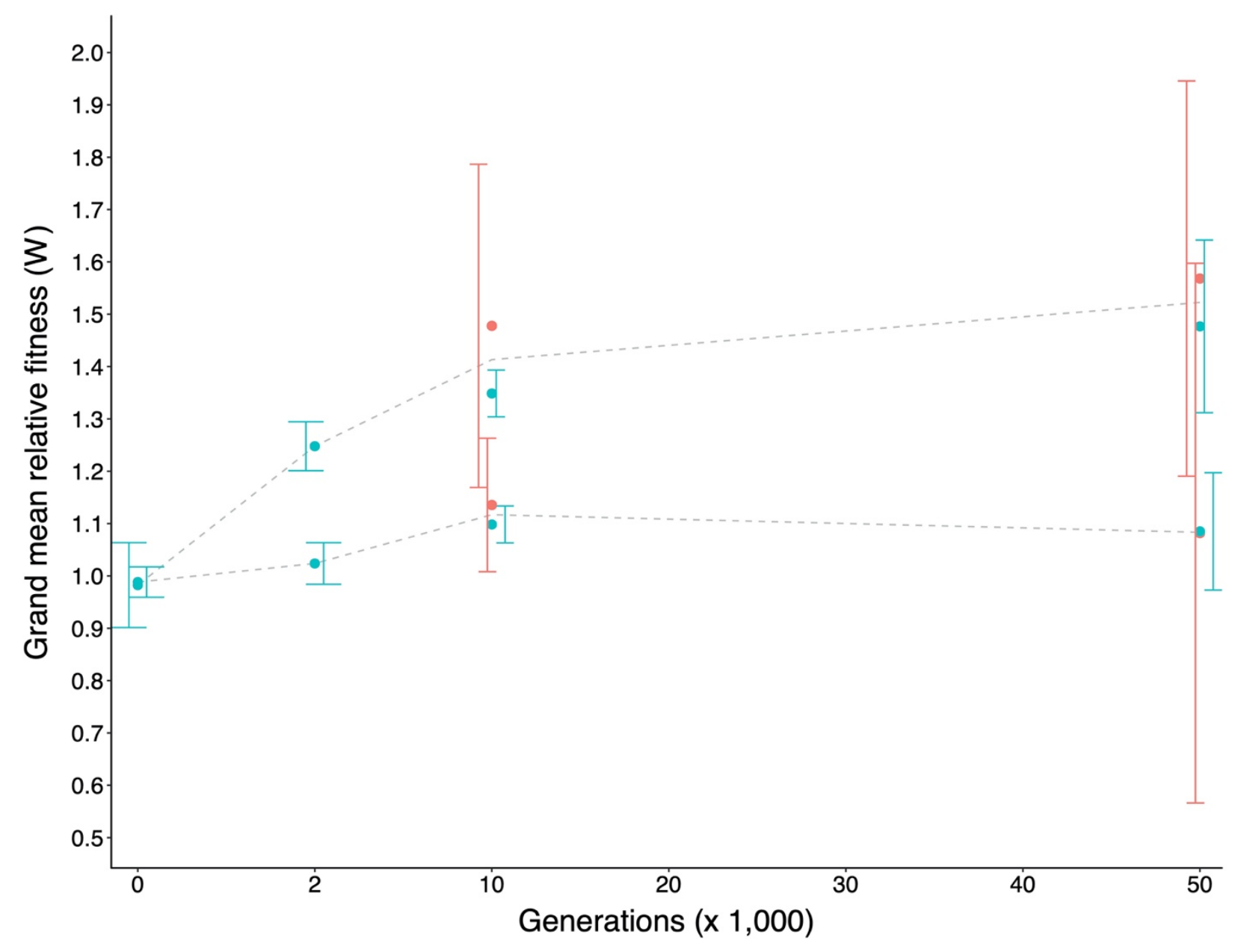

Figure S4: Relative fitness trajectories for the LTEE populations shown by their mutator status. Each point is the grand-mean fitness value of a set of evolved clones sampled at 2,000, 10,000, and 50,000 generations relative to the ancestors. Non-mutators and mutators are shown in teal and orange, respectively. The upper and lower trajectories were measured in the oxic and anoxic environments, respectively. Error bars are 95\% confidence intervals. 


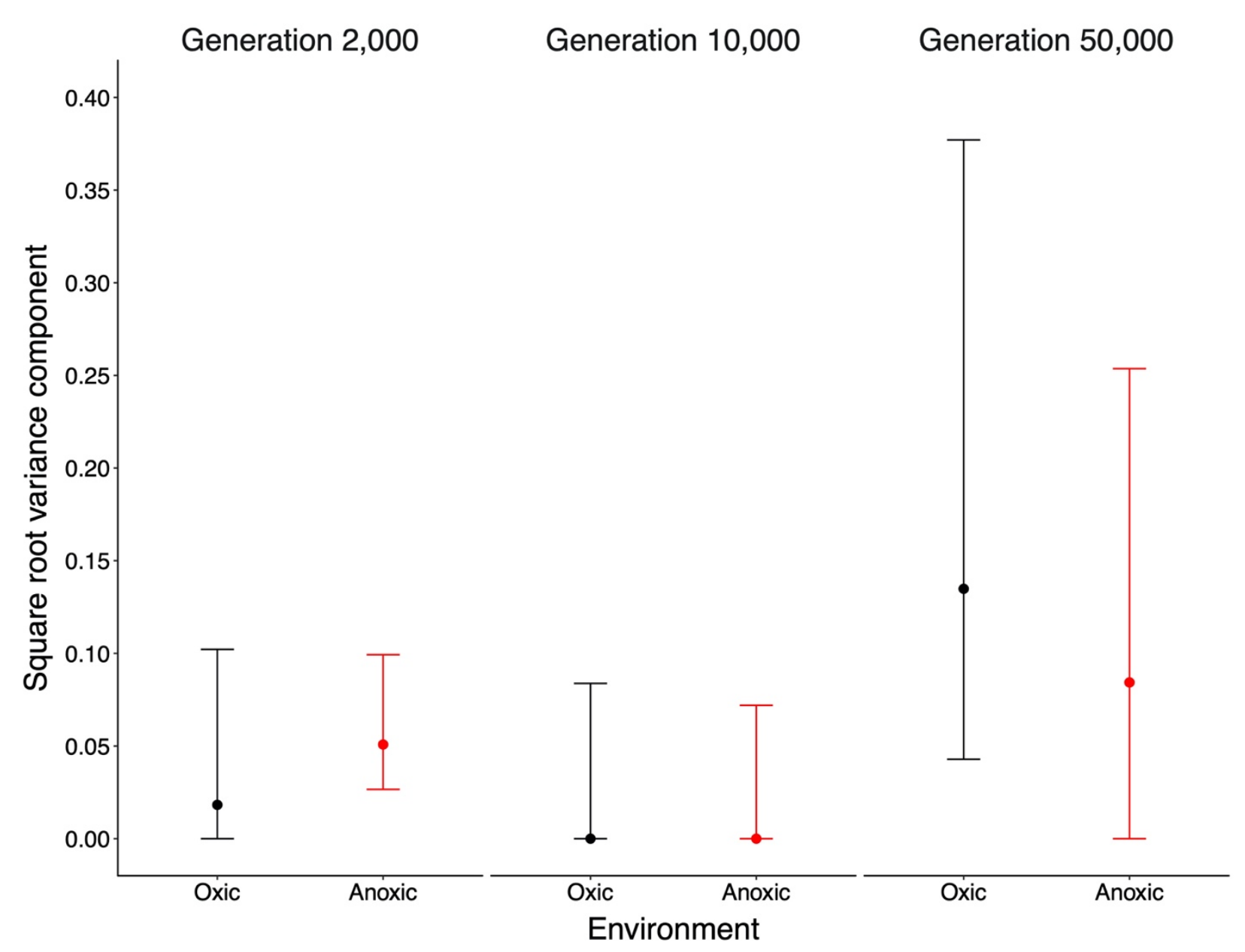

Figure S5: Among-population variance component for fitness, excluding mutator populations. See Figure 7 for additional details. The among-population variance is similar in the two environments whether the mutator populations are included (fig. 7) or excluded (this figure). 


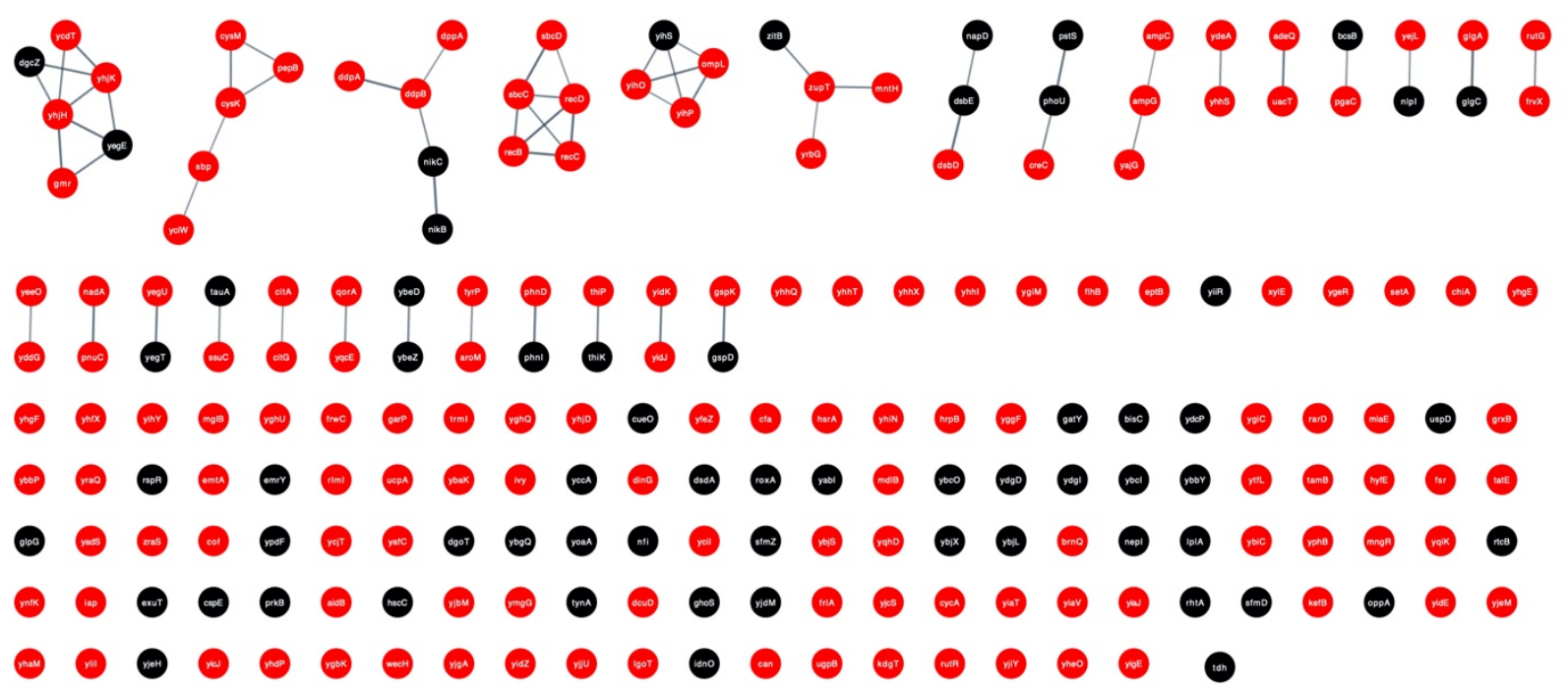

Figure S6: Network topology aerobic- (black) and anerobic-specific (red) genes that are not connected to the large core network shown in Figure 8. See the legend to that figure for additional details. 
Table S1: List of E. coli clones used in study

\begin{tabular}{ccc} 
Clone ID & Population & Generation \\
\hline REL606 & Ara $^{-}$ancestor & 0 \\
REL607 & Ara $^{+}$ancestor & 0 \\
REL1158A & Ara+1 & 2,000 \\
REL1159A & Ara+2 & 2,000 \\
REL1160A & Ara+3 & 2,000 \\
REL1161A & Ara+4 & 2,000 \\
REL1162A & Ara+5 & 2,000 \\
REL1163A & Ara+6 & 2,000 \\
REL1164A & Ara-1 & 2,000 \\
REL1165A & Ara-2 & 2,000 \\
REL1166A & Ara-3 & 2,000 \\
REL1167A & Ara-4 & 2,000 \\
REL1168A & Ara-5 & 2,000 \\
REL1169A & Ara-6 & 2,000 \\
REL4530A & Ara+1 & 10,000 \\
REL4531A & Ara+2 & 10,000 \\
REL4532A & Ara+3 & 10,000 \\
REL4533A & Ara+4 & 10,000 \\
REL4534A & Ara+5 & 10,000 \\
REL4535A & Ara+6 & 10,000 \\
REL4536A & Ara-1 & 10,000 \\
REL4537A & Ara-2 & 10,000 \\
REL4538A & Ara-3 & 10,000 \\
REL4539A & Ara-4 & 10,000 \\
REL4540A & Ara-5 & 10,000 \\
REL4541A & Ara-6 & 10,000 \\
REL11392 & Ara+1 & 50,000 \\
REL11342 & Ara+2 & 50,000 \\
REL11345 & Ara+3 & 50,000 \\
REL11348 & Ara+4 & 50,000 \\
REL11367 & Ara+5 & 50,000 \\
REL11370 & Ara+6 & 50,000 \\
REL11330 & Ara-1 & 50,000 \\
REL11333 & Ara-2 & 50,000 \\
REL11364 & Ara-3 & 50,000 \\
REL11336 & Ara-4 & 50,000 \\
REL11339 & Ara-5 & 50,000 \\
\hline & Ara-6 & 50,000 \\
\hline
\end{tabular}


Table S2: ANOVAs of relative fitness for clones sampled from non-mutator populations at three timepoints and measured in the oxic or anoxic environment

\begin{tabular}{|c|c|c|c|c|c|c|c|}
\hline \multirow[b]{2}{*}{ Generation } & \multirow[b]{2}{*}{ Source } & \multicolumn{3}{|c|}{ Oxic } & \multicolumn{3}{|c|}{ Anoxic } \\
\hline & & df & $F$ & $p$ & df & $\boldsymbol{F}$ & $p$ \\
\hline \multirow{2}{*}{2,000} & Lineage & 10 & 1.0441 & 0.4246 & 10 & 3.6004 & 0.0016 \\
\hline & Error & 43 & & & 42 & & \\
\hline \multirow{2}{*}{10,000} & Lineage & 7 & 0.5832 & 0.7644 & 7 & 0.7684 & 0.6180 \\
\hline & Error & 32 & & & 31 & & \\
\hline \multirow{2}{*}{50,000} & Lineage & 5 & 3.2700 & 0.0234 & 5 & 2.6544 & 0.0478 \\
\hline & Error & 22 & & & 24 & & \\
\hline
\end{tabular}

Note: Data were obtained for 11,8, and 6 lineages at 2,000, 10,000, and 50,000 generations, respectively. See Table 1 for additional details. 